\title{
Predictive Control for Interval Type-2 Fuzzy System with Event-Triggered Scheme
}

\author{
Siyao Wang, ${ }^{1}$ Xiaoming Tang $\mathbb{D}^{1,2}$ Li Deng, ${ }^{1}$ Hongchun Qu, \\ Linfeng Tian, ${ }^{3}$ and Cheng Tan ${ }^{4}$ \\ ${ }^{1}$ Chongqing University of Posts and Telecommunications, College of Automation, Chongqing 400035, China \\ ${ }^{2}$ Key Laboratory of Industrial Internet of Things \& Networked Control, Ministry of Education, \\ Chongqing University of Posts and Telecommunications, College of Automation, Chongqing 400035, China \\ ${ }^{3}$ Chongqing College of Electronic Engineering, Engineer of Network Center, Chongqing 400037, China \\ ${ }^{4}$ China Coal Technology and Engineering Group Chongqing Research Institute, Chongqing 400037, China
}

Correspondence should be addressed to Xiaoming Tang; txmmyeye@126.com

Received 26 February 2019; Revised 5 June 2019; Accepted 13 June 2019; Published 8 July 2019

Academic Editor: Antonin Dvorák

Copyright (C) 2019 Siyao Wang et al. This is an open access article distributed under the Creative Commons Attribution License, which permits unrestricted use, distribution, and reproduction in any medium, provided the original work is properly cited.

In this paper, a synthesis approach of model predictive control (MPC) is proposed for interval type-2 (IT2) Takagi-Sugeno (T-S) fuzzy system with quantization error, bounded disturbance, and data loss. The novelty lies in the following technical improvements. In order to reduce the redundant data transmission, an event-triggered communication scheme is applied to determine whether the control law should be transmitted into the communication network or not. The IT2 T-S fuzzy model is utilized to address the nonlinearity of plant with parameter uncertainties, which can be captured by the lower and upper membership functions. Furthermore, the phenomena of data loss and quantization error between the controller and the actuator are expressed as Markovian chain and sector-bound uncertainties. The synthesis approach of MPC is provided by solving an MPC optimization problem over an infinite horizon objective function which explicitly considers the input constraints. By applying the quadratic boundedness (QB) technique, the recursive feasibility and quadratic stability of closed-loop system can be guaranteed. A numerical simulation and comparison studies are proposed to illustrate the effectiveness of this approach.

\section{Introduction}

The networked control systems (NCSs) have played a more and more important role in many areas, which depend on the convenience of communication networks, such as low cost, simple installation and maintenance, and reduced system wiring [1-4]. However, communication networks also bring some challenges to the corresponding control system. The main problems are data loss and quantization error, which may degrade the control performance of NCSs. Caused by the limited capacities of communication networks, the signals always should be quantized before being transmitted into the network. Beyond that, owing to the unreliable features of communication networks, the data may be lost. Thus, the control performance of NCSs will be degraded by the quantization error and data loss inevitably. Many nice works about addressing data loss and quantization error problems under the framework of linear system theory are proposed in the past decades; see, e.g., [5-7].

Recently, the nonlinear characteristics of the NCSs have attracted a lot of attention owing to their practical applications. Takagi-Sugeno (T-S) fuzzy model is a popular method to address the nonlinearity of the NCSs, which bridges the gap between the complex nonlinear systems and linear systems [8]. Almost all the smooth nonlinear systems can be converted into linear systems by using T-S fuzzy model. Reference [9] proposed a new quadratic stabilization condition for the T-S fuzzy system, which based on the linear matrix inequalities (LMIs) technique. Reference [10] designed a method to address stability analysis and stabilization problems for the continuous T-S fuzzy system with timedelay. In [11], a new adaptive terminal sliding mode control method for single-input multi-output T-S fuzzy system with unknown and external disturbance was investigated. It is 
worth mentioned that there exist no uncertainties in the membership functions for the type-1 T-S fuzzy system; thus, the above researches based on the type-1 T-S fuzzy model may result in conservatism. To our delight, the IT2 T-S fuzzy model is imposed to deal with the parameter uncertainties of nonlinear systems captured by the lower and upper membership function [12-15]. In many aspects, IT2 T-S fuzzy model has better performance than the type-1 T-S model, which can be approved by some applications, such as DCDC converters [5], autonomous mobile robot [6], airplane flight control [16], and aerospace theory [17]. Moreover, many nice works have begun to design and analyze IT2 T$S$ fuzzy systems under network environment. In [18], the parameter uncertain in nonlinear networked control systems was described as lower and upper membership functions and relative weighting functions; furthermore, the phenomenon of data loss also has been taken into account. Reference [19] proposed an IT2 T-S fuzzy filter for nonlinear NCSs with data loss and quantization; beyond that, stochastic stability with $H_{\infty}$ performance can be guaranteed.

In NCSs, we often exploit time-triggered scheme; however, by this way, almost all data will be transmitted into the network in spite of whether the data is necessary or not. It is obvious that time-triggered scheme will reduce the efficiency of communication resources; see, e.g., [7, 20]. Event-triggered scheme can solve this problem well, which defined a trigger condition to determine whether the data should be transmitted into the network or not. By using this scheme, the burden of the network will be reduced effectively. In [21], an eventtriggered communication scheme was exploited to decide whether the data should be transmitted to the controller and achieve better utilization of network resources. In [22], by applying the event-triggered communication scheme and time-triggered periodic communication scheme for T-S fuzzy NCSs, respectively, it proved that the event-triggered scheme would utilize fewer communication resources while preserving the desired control performance. In [23], it considered the networked nonlinear systems with imperfect premise matching, which alleviated the burden of communication networks by using the event-triggered scheme. Reference [24] constructed a fuzzy observer with the imperfect premise matching to estimate the unmeasurable states of networked T-S fuzzy systems and addressed the problem of an eventtriggered nonparallel distribution compensation control to achieve higher communication efficiency and less conservation. It is apparently that the above results show the great advantages of event-triggered scheme. However, the state constraints and input constraints also exist in practical NCSs and have not been taken into account.

Model predictive control (MPC) is widely applied in industrial and academic communities since the defining feature of handling the physical constraints in a systematic manner [25-28]. At each sampling time, MPC is to solve a finite horizon optimization control problem based on the current measurements in order to obtain an optimal control sequence; however, only the first control of the optimal sequence is implemented. In the past decades, a great deal of researches [29-32] are based on the different branches of it, which have facilitated the development of this advanced method. Deserved to be mentioned, the synthesis approaches of MPC, as the important part of MPC, have attracted much attention and made some representative achievements; see $[33,34]$. Reference [33] proposed a novelty method for linear NCSs based on the classical synthesis approach of MPC, which specified the recursive feasibility and closed-loop stability; both of data loss and quantization error problems are considered in the transmission links. Reference [34] investigated the synthesis approach of MPC for the stochastic system by describing the data loss as Markovian model and the quantization error as bound uncertainties, which guaranteed the mean square stability and recursive feasibility simultaneously. However, neither of them considered releasing the burden of communication networks by using eventtriggered scheme.

In this paper, we discuss the synthesis approach of MPC for IT2 T-S fuzzy NCSs via event-triggered scheme. The controller output should be quantized before it is transmitted into the unreliable network, and the phenomenon of data loss and quantization error is taken into consideration simultaneously. In order to reduce the burden of communication networks, an event-triggered scheme is applied to decide whether the data should be released into the network or not. And in the transmission of triggered data, data loss and quantization error are expressed as Markovian chain and bound uncertain, respectively. An online MPC optimization problem that minimizes the upper bound of a quadratic objective function in an infinite time horizon subject to input constraints is proposed via the linear matrix inequality technique. Further, the recursive feasibility and closed-loop stability also can be guaranteed.

Notation. Throughout this paper, $I$ is an identity matrix with appropriate dimensions. $Q>0(\geq 0)$ represents $Q$ which is a real symmetric and positive-definite (semidefinite) matrix. $\|x\|_{Q}^{2}=x^{T} Q x$. In block symmetric matrices, the symbol $*$ is used to represent a term that is induced by symmetric and $\operatorname{diag}\{\cdots\}$ stands for block-diagonal matrix. $\Theta_{P}:=\left\{x \mid x^{T} P x \leq 1\right\}$ denotes the ellipsoid associated with the symmetric positive-definite matrix $P$. Co $\{\cdots\}$ denotes the convex combination of elements in $\{\cdot\}$, with the scalar combining coefficients nonnegative and their sum equal to 1. The notation $x(k+i \mid k)$ denotes future state of $x$ at time $k+i$. $\mathbb{E}$ is the expectation operator.

\section{Problem Formulation}

2.1. IT2 T-S Fuzzy Model and Controller. Consider a discretetime IT2 T-S fuzzy model, with $r$ th rules. It can be described as follows.

Plant Rule $i$. IF $f_{1}(p(k))$ is $M_{1}^{i}, f_{2}(p(k))$ is $M_{2}^{i}$ and $\cdots$ and $f_{\ell}(p(k))$ is $M_{\ell}^{i}$, THEN

$$
x(k+1)=A_{i} x(k)+B_{i} u(k)+D_{i} w(k)
$$

where $M_{a}^{i}$ represents the IT2 T-S fuzzy set of the function $f_{a}(p(k)), a=1,2, \ldots \ell ; i=1,2, \ldots, r . x(k) \in \mathfrak{R}^{n_{x}}$ is the state variable, $u(k) \in \mathfrak{R}^{n_{u}}$ is the input vector, $w(k) \in \mathfrak{R}^{n_{w}}$ is 
the persistent disturbance, and satisfied $w(k) \in \Theta_{P_{w}}$ and $A_{i}, B_{i}, D_{i}$ are constant matrices of appropriate dimensions. The firing strength of the $i$ th rule can be replaced by an interval sets as follows: $\widetilde{\varphi}_{i}(p(k))=\left[\varphi_{i}^{L}(p(k)), \varphi_{i}^{U}(p(k))\right]$, where $\varphi_{i}^{L}(p(k))=\prod_{a=1}^{\ell} \bar{\omega}_{M_{a}^{i}}\left(f_{a}(p(k))\right) \geq 0$, and $\varphi_{i}^{U}(p(k))=$ $\prod_{a=1}^{\ell} \underline{\omega}_{M^{i}}\left(f_{a}(p(k))\right) \geq 0$, which, respectively, denote the lower and upper grades of membership, and $\underline{\omega}_{M_{a}^{i}}\left(f_{a}(p(k))\right) \epsilon$ $[0,1], \bar{\omega}_{M_{a}^{i}}\left(f_{a}(p(k))\right) \in[0,1]$. Then, the IT2 T-S fuzzy model is inferred as follows:

$$
x(k+1)=\sum_{i=1}^{r} \omega_{i}(x(k))\left(A_{i} x(k)+B_{i} u(k)+D_{i} w(k)\right)
$$

where $\omega_{i}(p(k))=\omega_{i}^{L}(p(k)) \underline{e}_{i}(p(k))+\omega_{i}^{U}(p(k)) \bar{e}_{i}(p(k))$ and $\sum_{i=1}^{r} \omega_{i}(p(k))=1, \underline{e}_{i}(p(k)) \in[0,1]$, and $\bar{e}_{i}(p(k)) \in[0,1]$; both are nonlinear and satisfying $\underline{e}_{i}(p(k))+\bar{e}_{i}(p(k))=1$.

The IT2 T-S fuzzy controller with $r$ rules is denoted as follows: Controller Rule $j$ : IF $g_{1}(p(k))$ is $N_{1}^{j}, g_{2}(p(k))$ is $N_{2}^{j}$ and $\cdots$ and $g_{\ell}(p(k))$ is $N_{\alpha}^{j}$, THEN:

$$
v(k)=K_{j} x(k)
$$

where $N_{b}^{j}$ is the IT2 T-S fuzzy set of the function $g_{b}(p(k))$, $b=1,2, \ldots l ; j=1,2, \ldots, r . K_{j}$ is the feedback control law, and $v(k) \in \mathfrak{R}^{n_{v}}$ is the controller output. The firing strength of the $j$ th rule can be replaced by interval sets as follows: $\widetilde{\psi}_{j}(p(k))=\left[\psi_{j}^{L}(p(k)), \psi_{j}^{U}(p(k))\right]$, where $\psi_{j}^{L}(p(k))=\prod_{b=1}^{l} \underline{\zeta}_{N_{b}^{j}}\left(g_{b}(p(k))\right) \geq 0$ and $\psi_{j}^{U}(p(k))=$ $\prod_{b=1}^{\iota} \bar{\zeta}_{N_{b}^{j}}\left(g_{b}(p(k))\right) \geq 0$, which, respectively, denote the lower and upper grades of membership, and $\underline{\zeta}_{N_{b}^{j}}\left(g_{b}(x(k))\right) \epsilon$ $[0,1], \bar{\zeta}_{N_{b}^{j}}\left(g_{b}(x(k))\right) \in[0,1]$. Then, the IT2 T-S fuzzy model is inferred as follows:

$$
v(k)=\sum_{j=1}^{r} h_{j}(p(k)) K_{j} x(k)
$$

where $h_{j}(p(k))=\left(n_{j}^{L}(p(k)) \underline{h}_{j}(p(k))+n_{j}^{U}(p(k)) \bar{h}_{j}(p(k))\right) /$ $\left(\sum_{j=1}^{r}\left(n_{j}^{L}(p(k)) \underline{h}_{j}(p(k))+n_{j}^{U}(p(k)) \bar{h}_{j}(p(k))\right)\right), \quad$ and $\sum_{j=1}^{r} n_{j}(p(k))=1, \underline{h}_{j}(p(k)) \in[0,1]$ and $\bar{h}_{j}(p(k)) \in[0,1]$, both are nonlinear and satisfying $\underline{h}_{j}(p(k))+\bar{h}_{j}(p(k))=1$.

Moreover, hard constraints are always caused by physical constraints and imposed on the manipulated variables, which can be written as follows:

$$
\left|u_{l}(k)\right| \leq \bar{u}_{l}, \quad l=1, \ldots, m .
$$

2.2. Event-Triggered Communication Scheme. We focus on the system controller output data transmitted over a communication network. Figure 1 shows the framework of NCSs. In order to save the limited communication resource, an eventtriggered scheme is exploited to decide whether the data will be transmitted into network or not, i.e., comparing the current data with the last released data to determine whether

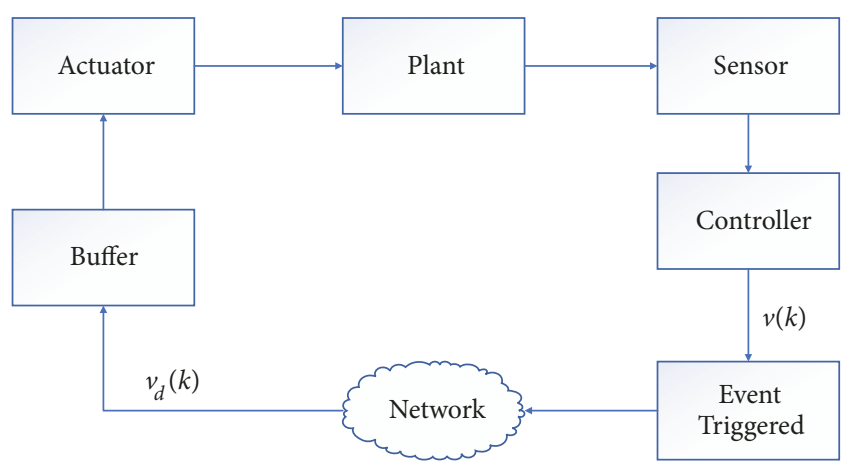

FIGURE 1: Framework of NCSs with event-triggered communication scheme.

$v(k)$ should be released. Define the error between the current data $v(k)$ and the last released data $\widetilde{v}\left(i_{k}\right)$ as $\phi(k)=v(k)-\widetilde{v}\left(i_{k}\right)$, where the $k \in\left[i_{k}, i_{k+1}\right)$ is the current time, $i_{k}$ is the last eventtriggered time, and $i_{k+1}$ is the next event-triggered time. In trigger time, $d_{i_{k}}=k-i_{k}-1$, where $d_{i_{k}}$ represents the ignored data between $i_{k}$ and $k$. If the event-triggered condition can be satisfied, the data $v(k)$ will be transmitted into the unreliable network and become a new latest event-triggered data; else, the last released data will be maintained and the current data will be ignored. The next event-triggered condition is inferred as

$$
\|\phi(k)\|_{Q}^{2} \geq \eta\left\|v_{i_{k}}\right\|_{Q}^{2}
$$

where $\eta$ is given scalar and $\eta \in[0,1]$; $Q$ is a positive-definite matrix with appropriate dimension. We define two sequence $B_{1}=\{-1,0,1,2, \ldots, k, \ldots\}$ and $B_{2}=\left\{i_{0}, i_{1}, i_{2}, \ldots, i_{k}, \ldots\right\}$ to describe the event-triggered scheme more specifically. The $B_{1}$ is the sequence of controller output $v(k)$ and $B_{2}$ is the eventtriggered sequence. It is apparent that $B_{1} \in B_{2}$, if all the data are triggered, $B_{1}=B_{2}$.

When the data is transmitted into the unreliable network, the data loss from the controller to the actuator occurs inevitably. We define a stochastic variable $\gamma(k) \in\{0,1\}$ to denote the state of time $k, \gamma=1$ means data is transmitted successfully, and $\gamma=0$ means missing data. It is assumed that the phenomenon of data loss satisfies a discrete-time homogeneous Markov chain. The data recovery probability and the data loss probability are $\beta$ and $\alpha$, respectively, where $\alpha=$ $\operatorname{Pr}(\gamma(k+1)=0 \mid \gamma(k)=1)$ and $\beta=\operatorname{Pr}(\gamma(k+1)=1 \mid \gamma(k)=0)$. The corresponding transition probability matrix is defined as follows:

$$
\Omega=\left[\begin{array}{cc}
1-\beta & \beta \\
\alpha & 1-\alpha
\end{array}\right]
$$

It is obvious that only the event-triggered data will be lost in the network, which means data will be lost in sequence $B_{2}$. A compensation strategy is proposed to resolve the problems of missing data. If the data are transmitted successfully, $v_{d}(k)=\widetilde{v}(k)$. If the data are missed, $v_{d}(k)=\theta v_{d}(k-1)$ and $v_{d}(k-1)$ is the last released 
data at time $k-1$, where the $\theta(\theta \in[0,1])$ is a forgetting factor. We assume that the upper of the data loss is $\vartheta_{\max }$. For further processing and simplifying the exposition, we define $\left\{\varrho\left(i_{k}\right)\right\}=\left\{\varrho\left(i_{0}\right), \varrho\left(i_{1}\right), \varrho\left(i_{2}\right), \ldots, \varrho\left(i_{k}\right), \ldots\right\}$; the sequence received by the buffer is $\left\{\varrho\left(i_{k}\right) \widetilde{v}_{i_{k}}\right\}=$ $\left\{\varrho\left(i_{0} \widetilde{v}_{i_{0}}\right), \varrho\left(i_{1}\right) \widetilde{v}_{i_{1}}, \varrho\left(i_{2}\right) \widetilde{v}_{i_{2}}, \ldots, \varrho\left(i_{k}\right) \widetilde{v}_{i_{k}}, \ldots\right\}$.

Due to the effectiveness of the buffer and $d_{i_{k}}=k-i_{k}-1$, the output of the buffer is denoted as $\{\widetilde{v}(k)\}=$ $\{\underbrace{\bar{v}\left(i_{0}\right), \ldots, \bar{v}\left(i_{0}\right)}_{i_{i_{0}}+1}, \underbrace{\bar{v}\left(i_{1}\right), \ldots, \bar{v}\left(i_{1}\right)}_{d_{i_{1}}+1}, \ldots, \underbrace{\bar{v}\left(i_{k}\right), \ldots, \bar{v}\left(i_{k}\right)}_{d_{i_{k}}+1}, \ldots\}$.

Thus, we define a new random sequence to represent the data received by the actuator $\{\gamma(k)\}=$ $\{\underbrace{\gamma\left(i_{0}\right), \ldots, \gamma\left(i_{0}\right)}_{i_{i_{0}}+1}, \underbrace{\gamma\left(i_{1}\right), \ldots, \gamma\left(i_{1}\right)}_{d_{i_{1}}+1}, \ldots, \underbrace{\gamma\left(i_{k}\right), \ldots, \gamma\left(i_{k}\right)}_{i_{i_{k}}+1}, \ldots\}$.

Therefore, we can concluded

$$
\begin{aligned}
& v_{d}(k)=\gamma(k) \widetilde{v}_{i_{k}}+(1-\gamma(k)) \theta v_{d}(k-1), \\
& k \in\left[i_{k}, i_{k+1}\right)
\end{aligned}
$$

Considering $k \in\left[i_{k}, i_{k+1}\right)$, we have $\widetilde{v}_{i_{k}}=v(k)-\phi(k)$. Then, we can obtain

$$
v_{d}(k)=\gamma(k)(v(k)-\phi(k))+(1-\gamma(k)) \theta v_{d}(k-1)
$$

Remark 1. In order to avoid the particularity, it is assumed that $v(-1)$ is triggered; i.e., $\widetilde{v}\left(i_{0}\right)=v(-1)$. It is worth mentioning that the $\eta$ is inversely proportional to the eventtriggered frequency.

Remark 2. The forgetting factor is changeable so that it can compensate for the data loss better. When the $\theta=0$, it means no compensation, which also represents the zeroinput between the controller to the actuator when the data loss occurs. $\theta=1$ means the latest transmitted successfully data will be transmitted into the actuator when the current data is lost.

2.3. Quantization Scheme. The quantizer model has the following form:

$$
u(k)=f(\xi(k))
$$

where $f(\xi)$ denotes the scalar input $\xi \in R$ under the logarithmic quantizer condition, which can be inferred as

$$
f(\xi)= \begin{cases}\mu_{i}, & \text { if } \frac{1}{1+\delta} \mu_{i}<\xi<\frac{1}{1-\delta} \mu_{i}, \xi>0 \\ 0, & \text { if } \xi=0 \\ -f(-\xi), & \text { if } \xi<0\end{cases}
$$

where $\delta=(1-\rho) /(1+\rho), 0<\rho<1$. The quantized level is described as $\chi=\left\{ \pm \mu_{i}, \mu_{i}=\rho^{i} \mu_{0}, i= \pm 1, \pm 2, \ldots\right\} \cup$ $\mu_{0} \cup\{0\}, \mu_{0}>0$. Every quantization level $\mu_{i}$ corresponds to a segment of $\xi$ so that the quantizer can maps the whole segment of $\xi$. A research [35] proposed a sectorbound approach, which represented the every value of $f(\cdot)$ by $f(\xi)=(I+\varpi) \xi$, where $₫ \in([-\delta, \delta]$. In this paper, $\xi(k)$ can be choose as $v_{d}(k)$, so the control input from the quantizer $u(k)$ can be written as

$$
\begin{aligned}
u(k) & =f(v(k)) \\
& =\left[f_{1}\left(v_{1}(k)\right) f_{2}\left(v_{2}(k)\right) \cdots f_{m}\left(v_{m}(k)\right)\right] \\
& =\Lambda(k) v(k)
\end{aligned}
$$

where $\Lambda(k)=\operatorname{diag}\left\{1+\Phi_{1}(k), 1+\Phi_{2}(k), \ldots, 1+\emptyset\right\} \in$ $\Xi=\operatorname{Co}\left\{\Lambda^{(1)}, \Lambda^{(2)}, \ldots, \Lambda^{\left(2^{m}\right)}\right\}, \omega_{j}(k)=\left[-\delta_{p}, \delta_{p}\right], p=$ $1, \ldots, m, \Lambda^{q}$ is a diagonal matrix, which consists of $1-\delta_{p}$ or $1+\delta_{p}$.

Based on (2), (4), (9), and (12), the closed-loop dynamic model (4) can be written as follows:

$$
\begin{aligned}
& x(k+1)=\sum_{i=1}^{r} \sum_{j=1}^{r} \omega_{i}(p(k)) h_{j}(p(k)) \\
& \cdot\left\{\left(A_{i}+\gamma(k) B_{i} \Lambda(k) K_{i j}\right) x(k)\right. \\
& +(1-\gamma(k)) B_{i} \theta v_{d}(k-1)-\gamma(k) B_{i} \phi(k) \\
& \left.+D_{i} w(k)\right\} \\
& u(k)=\sum_{i=1}^{r} \sum_{j=1}^{r} \omega_{i}(p(k)) h_{j}(p(k))\left\{\gamma(k) \Lambda(k) K_{i j} x(k)\right. \\
& \left.\left.+(1-\gamma(k)) \theta v_{d}(k-1)\right)-\gamma(k) \phi(k)\right\}
\end{aligned}
$$

Then, let $z(k)=\left[\begin{array}{ll}x^{T}(k) & v_{d}^{T}(k-1)\end{array}\right]^{T}, \widetilde{z}(k)=$ $\left[\begin{array}{lll}z^{T}(k) & \phi^{T}(k) & w^{T}(k)\end{array}\right]^{T}$, according to the (13) and (14), the augmented model is denoted as

$$
z(k+1)=\bigwedge(p(k)) \Upsilon_{1} \tilde{z}(k)
$$

where $\bigwedge(p(k))=\sum_{i=1}^{r} \sum_{j=1}^{r} \omega_{i}(p(k)) h_{j}(p(k))$,

$$
\begin{aligned}
& \Upsilon_{1} \\
& =\left[\begin{array}{cccc}
A_{i}+\gamma(k) B_{i} \Lambda(k) K_{j} & (1-\gamma(k)) \theta B_{i} & -\gamma(k) B_{i} & D_{i} \\
\gamma(k) \Lambda(k) K_{i j} & (1-\gamma(k)) \theta & -\gamma(k) & 0
\end{array}\right]
\end{aligned}
$$

Remark 3. Consider the multiple data loss; if continuous data loss occurred $d$ times, $0<d \leq \vartheta_{\max }, v=\theta^{d} v(k-d)$.

If the closed-loop system in every initial state $x(0)$ satisfies $E_{x(0)}\left\{\sum_{k=0}^{\infty} x^{T}(k) x(k)\right\}<\infty$, then we can consider the system is stochastically stable. 


\section{Main Results}

3.1. Event-Triggered State-Feedback MPC. In this part, a novel event-triggered constrained MPC for IT2 T-S fuzzy system with Markovian data loss method is developed. Define the performance objective function as $J_{\infty}(k)=\sum_{i=0}^{\infty} \mathbb{E} \| z(k+i \mid$ $k)\left\|_{S}^{2}+\right\| u(k+i \mid k) \|_{R}^{2}, S>0, R>0$ with suitable dimension, and follow quadratic Lyapunov function

$$
V(k+i \mid k)=z^{T}(k+i \mid k) \Delta(k+i \mid k) z(k+i \mid k)
$$

where $\Delta(k+i \mid k)=\operatorname{diag}\left\{M_{\gamma(k+i \mid k)}, N_{\gamma(k+i \mid k)}\right\}, M_{\gamma(k+i \mid k)} \in$ $R^{m \times m}$ and $N_{\gamma(k+i \mid k)} \in R^{n \times n}$ are positive-definite matrix. $\gamma(k+$ $i \mid k) \in\{0,1\}$. For further discussion, we introduce the Lemma 4, which also can called QB technique. This method is applied to deal with the state estimation and bounded disturbance problems.

Lemma 4 (see [36] ). For all allowable $w(k+i \mid k), i \geq 0$, system (11) is quadratically bounded if

(i) $M(k+i \mid k), N(k+i \mid k)$ is Lyapunov matrix;

(ii) $\widetilde{z}(k)^{T} \Delta(k+i \mid k) \widetilde{z}(k) \geq 1$ implies that $\widetilde{z}(k+1)^{T} \Delta(k+i \mid$ $k) \widetilde{z}(k+1) \geq 1$;

(iii) the ellipsoid $\Theta_{\Delta(k+i \mid k)}$ is a positively invariant set.

The target is to design a state-feedback model predictive controller to solve the following optimization problem at each time $k$ :

$$
\begin{array}{rl}
\min _{\varepsilon, M, N, K_{i j}} & \mathcal{E} \\
\text { s.t. } \quad & \mathbb{E}\left\{\|z(k+i \mid k)\|_{\Delta(k+i \mid k)}^{2}\right\} \geq 1 \Longrightarrow \\
& \mathbb{E}\left\{\|z(k+i \mid k)\|_{\Delta(k+i \mid k)}^{2}\right. \\
& \left.-z(k+i+1 \mid k) \|_{\Delta(k+i \mid k)}^{2}\right\} \\
\geq \mathbb{E}\left\{\|z(k+i \mid k)\|_{S}^{2}+\|u(k+i \mid k)\|_{R}^{2}\right\} & -\bar{u} \leq u(k+i \mid k) \leq \bar{u} \\
& z(k) \in \Theta_{\Delta(k+i \mid k)}
\end{array}
$$

Assume $\lim _{i=\infty} \mathbb{E}\{z(k+i \mid k)\}=0$, then, summing (20) from $i=0$ to $i=\infty$, we can obtain $J_{\infty}(k) \leq V(k \mid k)$. Thus, the optimization problem is to minimize the upper in each step time $k$. The MPC optimization problem can be solved indirectly, which is noted as

$$
\begin{array}{ll}
\min & \varepsilon, \\
\text { s.t. } & V(k \mid k) \leq \varepsilon
\end{array}
$$

Deserved to be mentioned, whether the data is lost or not, we can assume the $u(k)$ at time $k$ can be transmitted. Therefore, we can obtain $V(k \mid k) \leq \varepsilon$; by Schur complement we have

$$
\left[\begin{array}{cc}
1 & * \\
z(k) & \bar{U}_{1}
\end{array}\right] \geq 0
$$

where $\bar{U}_{1}=\operatorname{diag}\left\{\bar{M}_{1}, \bar{N}_{1}\right\}, \bar{M}_{1}=\varepsilon M_{1}^{-1}, \bar{N}_{1}=\varepsilon N_{1}^{-1}$.
Then, the optimization problem with LMIs constraints will be shown as follows.

Theorem 5. Condition (19) and (20) can be satisfied under condition (25)-(28), if there exist scalars $\varepsilon$, matrices $L_{i j}$, and symmetric matrices $\left\{\overline{M_{0}}, \overline{M_{1}}, \overline{N_{0}}, \overline{N_{1}}, \overline{E_{0}}, \overline{E_{1}}, \overline{F_{0}}, \overline{F_{1}}, \bar{Q}\right\}$ satisfying the following conditions, where $L_{i j}=K_{i j} M_{1}^{-1}$. For simplicity, for a matrices $\mathscr{M}, \bar{M}=\varepsilon \mathscr{M}, \mathscr{M} \epsilon$ $\left\{M_{0}, M_{1}, N_{0}, N_{1}, E_{0}, E_{1}, F_{0}, F_{1}, Q\right\}$,

$$
\begin{aligned}
& \bigwedge(p(k))\left[\begin{array}{ll}
\Xi_{0} & * \\
\Xi_{1}^{q} & \Xi_{2}
\end{array}\right] \geq 0, \quad q=1, \ldots, 2^{m}, \\
& \bigwedge(p(k))\left[\begin{array}{ll}
U_{3} & * \\
\Xi_{3} & U_{2}
\end{array}\right] \geq 0, \\
& \bigwedge(p(k))\left[\begin{array}{ll}
\Xi_{4} & * \\
\Xi_{5}^{q} & \Xi_{6}
\end{array}\right] \geq 0, \quad q=1, \ldots, 2^{m}, \\
& \bigwedge(p(k))\left[\begin{array}{ll}
U_{4} & * \\
\Xi_{7} & U_{2}
\end{array}\right] \geq 0,
\end{aligned}
$$

where $q=1, \ldots, 2^{m}$,

$$
\begin{aligned}
& \Xi_{0}=\operatorname{diag}\left\{(1-\kappa) \overline{M_{1}},(1-\kappa) \overline{N_{1}}, \bar{Q}, \kappa P_{w}\right\}, \\
& \Xi_{2}=\operatorname{diag}\left\{\overline{E_{1}}, \overline{F_{1}}, \varepsilon S^{-1}, \varepsilon R^{-1}, \bar{Q}\right\}, \\
& U_{3}=\operatorname{diag}\left\{\overline{E_{1}}, \overline{F_{1}}\right\} \text {, } \\
& U_{2}=\operatorname{diag}\left\{\overline{M_{0}}, \overline{M_{1}}, \overline{N_{0}}, \overline{N_{1}}\right\} \text {, } \\
& \Xi_{4}=\operatorname{diag}\left\{(1-\kappa) \overline{M_{0}},(1-\kappa) \overline{N_{0}}, \bar{Q}, \kappa P_{w}\right\} \text {, } \\
& \Xi_{6}=\operatorname{diag}\left\{\overline{E_{0}}, \overline{F_{0}}, \varepsilon S^{-1}, \varepsilon R^{-1}, \bar{Q}\right\}, \\
& U_{4}=\operatorname{diag}\left\{\overline{E_{1}}, \overline{F_{1}}\right\} \\
& \Xi_{1}^{q}=\left[\begin{array}{cccc}
A_{i} \overline{M_{1}}+B_{i} \Lambda^{q} L_{i j} & 0 & \varepsilon D_{i} & -B_{i} \bar{Q} \\
L_{i j} \Lambda^{q} & 0 & 0 & -\bar{Q} \\
\overline{M_{1}} & 0 & 0 & 0 \\
L_{i j} \Lambda^{q} & 0 & 0 & -\bar{Q} \\
\eta^{1 / 2} \Lambda^{q} L_{i j} & 0 & 0 & -\eta^{1 / 2} \bar{Q}
\end{array}\right], \\
& \Xi_{3}=\left[\begin{array}{cc}
\alpha^{1 / 2} \overline{E_{1}} & 0 \\
\left(1-\alpha^{1 / 2} \overline{E_{1}}\right. & 0 \\
0 & \alpha^{1 / 2} \overline{F_{1}} \\
0 & \left(1-\alpha^{1 / 2}\right) \overline{F_{1}}
\end{array}\right] \text {, }
\end{aligned}
$$




$$
\begin{aligned}
& \Xi_{5}^{q}=\left[\begin{array}{cccc}
A_{i} \overline{M_{0}} & \theta B_{i} \overline{N_{0}} & \varepsilon D_{i} & 0 \\
0 & \theta \overline{N_{0}} & 0 & 0 \\
\overline{M_{0}} & 0 & 0 & \\
0 & \theta \overline{N_{0}} & 0 & 0 \\
\eta^{1 / 2} \Lambda^{q} L_{i j} & 0 & 0 & -\eta^{1 / 2} \bar{Q}
\end{array}\right], \\
& \Xi_{7}=\left[\begin{array}{cc}
\left(1-\beta^{1 / 2}\right) \overline{E_{0}} & 0 \\
\beta^{1 / 2} \overline{E_{0}} & 0 \\
0 & \left(1-\beta^{1 / 2}\right) \overline{F_{0}} \\
0 & \beta^{1 / 2} \overline{F_{0}}
\end{array}\right] .
\end{aligned}
$$

Proof. Since $\|w(k+i \mid k)\|_{P_{w}}^{2} \leq 1$, thus, we have $V(z(k+$ $i \mid k)) \geq 1$ is equivalent to $V(z(k+i \mid k)) \geq \| w(k+i \mid$ $k) \|_{P_{w}}^{2}$. Consider the event-triggered condition (10); when $k \in$ $\left[i_{k}, i_{k+1}\right)$, according the features of event-triggered scheme, we can obtain $\|\phi(k)\|_{Q}^{2} \leq \eta\left\|u_{i k}\right\|_{Q}^{2}$. Then we have the following form to satisfy condition (20)

$$
\begin{gathered}
\mathbb{E}\{\| V(k+i \mid k)\} \geq\|w(k+i \mid k)\|_{P_{w}}^{2} \Longrightarrow \\
\mathbb{E}\{V(k+i \mid k)-V(k+i+1 \mid k)\} \\
\geq-\mathbb{E}\left\{\|z(k+i \mid k)\|_{S}^{2}+\|u(k+i \mid k)\|_{R}^{2}\right\} \\
-\mathbb{E}\left\{\|u(k)-\phi(k)\|_{Q}^{2}-\|\phi(k)\|_{Q}^{2}\right\}
\end{gathered}
$$

Combining augmented closed-loop system (15) then we can obtain

$$
\begin{aligned}
& \widetilde{z}^{T}(k+i \mid k) \Delta_{\gamma(k+i \mid k)}^{1} \widetilde{z}^{T}(k+i \mid k) \geq 0 \Longrightarrow \\
& \widetilde{z}^{T}(k+i \mid k)\left\{\Delta_{\gamma(k+i \mid k)}^{2}-\Gamma^{T} \Delta_{\gamma(k+i+1 \mid k)}^{2} \Gamma-\operatorname{diag}\{S, 0,0\}\right. \\
& \left.\quad-\Gamma_{2}^{T} R \Gamma_{2}-\eta \Gamma_{3}^{T} Q \Gamma_{3}\right\} \widetilde{z}^{T}(k+i \mid k) \geq 0
\end{aligned}
$$

where

$$
\begin{aligned}
& \Gamma=\left[\begin{array}{cccc}
A_{i}+\gamma(k+i \mid k) B_{i} \Lambda(k+i \mid k) K_{j} & (1-\gamma(k+i \mid k)) \theta B_{i} & -\gamma(k+i \mid k) B_{i} & D_{i} \\
\gamma(k+i \mid k) \Lambda(k+i \mid k) K_{j} & (1-\gamma(k+i \mid k)) \theta & -\gamma(k+i \mid k) & 0
\end{array}\right] \\
& \Delta_{\gamma(k+i \mid k)}^{1}=\operatorname{diag}\left\{M_{\gamma(k+i \mid k)}, N_{\gamma(k+i \mid k)}, 0,-P_{w}\right\} \\
& \Delta_{\gamma(k+i \mid k)}^{2}=\operatorname{diag}\left\{M_{\gamma(k+i \mid k)}, N_{\gamma(k+i \mid k)}, 0, Q\right\} \\
& \Delta_{\gamma(k+i+1 \mid k)}^{2} \operatorname{diag}\left\{M_{\gamma(k+i \mid k) \mid \gamma(k+i+1 \mid k)}, N_{\gamma(k+i \mid k) \mid \gamma(k+i+1 \mid k)}, 0,0\right\} \\
& \Gamma_{2}=\left[\Lambda(k+i \mid k) K_{i j}(1-\gamma(k+i \mid k)) \theta \quad 0 \quad-\gamma(k+i \mid k)\right] \\
& \Gamma_{3}=\left[\begin{array}{llll}
K_{i j} & 0 & 0 & -1
\end{array}\right]
\end{aligned}
$$

Applying the S-procedure, (15) is satisfied for any possible $z(k+i)$ and $w(k+i)$, if and only if there exists a scalar $\kappa \in\{0,1\}$, such that

$$
\begin{aligned}
& \left\{\Delta_{\gamma(k+i \mid k)}^{2}-\Gamma^{T} \Delta_{\gamma(k+i+1 \mid k)}^{2} \Gamma-\operatorname{diag}\{S, 0,0\}-\Gamma_{2}^{T} R \Gamma_{2}\right. \\
& \left.-\eta \Gamma_{3}^{T} Q \Gamma_{3}\right\} \geq \kappa \Delta_{\gamma(k+i \mid k)}^{1}
\end{aligned}
$$

Consider the $\gamma(k+i \mid k)=1$ and $\mathbb{E}_{\gamma(k+i \mid k)}\left\{\Delta_{\gamma(k+i+1 \mid k)}\right\}=$ $\alpha \operatorname{diag}\left\{M_{0}, N_{0}\right\}+(1-\alpha) \operatorname{diag}\left\{M_{1}, N_{1}\right\}$, then we have the following inequalities:

$$
\begin{aligned}
\Gamma_{1}^{T} & {\left[\begin{array}{cc}
E_{1} & 0 \\
0 & F_{1}
\end{array}\right] \Gamma_{1} } \\
& -\operatorname{diag}\left\{(1-\kappa) M_{1},(1-\kappa) N_{1}, Q, \kappa P_{w}\right\} \\
& \times+\operatorname{diag}\{S, 0,0\}+\left[\Lambda(k+i \mid k) K_{i j} \quad 0 \quad 0 \quad-1\right]^{T} R
\end{aligned}
$$

$$
\begin{aligned}
& \times\left[\Lambda(k+i \mid k) K_{i j} \quad 0 \quad 0-1\right] \\
& +\eta^{1 / 2}\left[\begin{array}{llll}
K_{i j} & 0 & 0 & -1
\end{array}\right]^{T} \\
& \times Q\left[\begin{array}{llll}
K_{i j} & 0 & 0 & -1
\end{array}\right] \eta^{1 / 2} \leq 0
\end{aligned}
$$$$
\alpha \operatorname{diag}\left\{M_{0}, N_{0}\right\}
$$$$
+(1-\alpha) \operatorname{diag}\left\{M_{1}, N_{1}\right\} \leq \operatorname{diag}\left\{E_{1}, F_{1}\right\}
$$

where

$$
\Gamma_{1}=\left[\begin{array}{cccc}
A_{i}+B_{i} \Lambda(k+i \mid k) K_{j} & 0 & -B_{i} & D_{i} \\
\Lambda(k+i \mid k) K_{j} & 0 & -1 & 0
\end{array}\right]
$$

By pre- and postmultiplying $\operatorname{diag}\left\{E_{1}^{-1}, F_{1}^{-1}\right\}$ and $\operatorname{diag}\left\{\varepsilon^{1 / 2} M_{1}^{-1}, \varepsilon^{1 / 2} N_{1}^{-1}, \varepsilon^{1 / 2} Q_{1}^{-1}, \varepsilon^{1 / 2}\right\}$ and its transpose on both sides of (28) and (27), respectively, and then, by applying Schur complement, we can obtain (23) and (24). When $\gamma(k+i \mid k)=0$, using the similar approach we can 
get (25) and (26). If (27) and (28) hold, (20) can be satisfied apparently.

Theorem 6. Assuming the data transmitted successfully at time $k$, then at next time $k+1$, if the data is lost in transitions, according to the compensation strategy, it is obvious that the input constraints (5) are satisfied. But, when the data is transmitted successfully, we need to guarantee the invariant set condition (22). In fact, the invariant set condition can be satisfied if there exist symmetric matrices $\left\{\bar{M}_{0 . c}, \bar{N}_{0 . c}\right\}$, satisfying the following conditions, where $\bar{M}_{0 . c}, \bar{N}_{0 . c},(c=$ $\left.1, \ldots, \vartheta_{\max }\right)$.

$$
\begin{gathered}
\bigwedge(p(k))\left[\begin{array}{cc}
\bar{U}_{1} & * \\
\Xi_{8}^{q T} & \Xi_{0}^{1}
\end{array}\right] \geq 0, \quad q=1, \ldots, 2^{m}, \\
\bigwedge(p(k))\left[\begin{array}{cc}
\bar{U}_{0,1} & * \\
\Xi_{8}^{q T} & \Xi_{0}^{1}
\end{array}\right] \geq 0, \quad q=1, \ldots, 2^{m}, \\
\bigwedge(p(k))\left[\begin{array}{cc}
\Xi_{0, c} & * \\
\Xi_{0, c}^{1} & \bar{U}_{1}
\end{array}\right] \geq 0, \quad s=1, \ldots, \vartheta_{\max }, \\
\bigwedge(p(k))\left[\begin{array}{cc}
\bar{U}_{0, c+1} & * \\
\Xi_{0, c}^{1} & \Xi_{0, c}
\end{array}\right] \geq 0, \quad s=1, \ldots, \vartheta_{\max }-1,
\end{gathered}
$$

where

$$
\begin{aligned}
& \Xi_{0}^{1}=\operatorname{diag}\left\{\bar{M}_{1}, \bar{N}_{1}, \bar{Q}, D_{i} P_{w}\right\}, \\
& \Xi_{8}^{q}=\left[\begin{array}{cccc}
A_{i} \bar{M}_{1}+B_{i} L_{i j} \Lambda^{q} & 0 & -\bar{Q} B_{i} & D_{i} P_{w} \\
L_{i j} \Lambda^{q} & 0 & -\bar{Q} & 0
\end{array}\right], \\
& \Xi_{0, c}^{1}=\left[\begin{array}{cccc}
A_{i} \bar{M}_{0, c} & \theta B_{i} \bar{N}_{0, s} & 0 & D_{i} P_{w} \\
0 & \theta B_{i} \bar{N}_{0, c} & 0 & 0
\end{array}\right] \text {, } \\
& \bar{U}_{0,1}=\operatorname{diag}\left\{\bar{M}_{0,1}, \bar{N}_{0,1}\right\} \text {, } \\
& \Xi_{0, c}=\operatorname{diag}\left\{\bar{M}_{0, c}, \bar{N}_{0, c}, \bar{Q}, D_{i} P_{w}\right\} \text {, } \\
& \bar{U}_{0, c+1}=\operatorname{diag}\left\{\bar{M}_{0, c+1}, \bar{N}_{0, c+1}, \bar{Q}, P_{w}\right\} .
\end{aligned}
$$

Proof. Assuming the data transmitted successfully at time $k$, the data can be transmitted from the controller to the actuator successfully. At time $k+1$, if the data lost, that is $\gamma(k \mid k)=0$, constraints (5) can be satisfied obviously; thus, the only case that should be analyzed is $\gamma(k+i \mid k)=1$. From the augmented closed-loop system (15), we can obtain

$$
\begin{aligned}
& {\left[\begin{array}{cccc}
A_{i}+B_{i} \Lambda(k) K_{j} & 0 & -B_{i} & D_{i} \\
\Lambda(k) K_{j} & 0 & -1 & 0
\end{array}\right] \Xi_{0}^{1^{-1}}} \\
& \times\left[\begin{array}{cccc}
A_{i}+B_{i} \Lambda(k) K_{j} & 0 & -B_{i} & D_{i} \\
\Lambda(k) K_{j} & 0 & -1 & 0
\end{array}\right]^{T} \leq \bar{U}_{1}^{-1}
\end{aligned}
$$

that is, $z(k+1 \mid k) \in \Theta_{\Delta(k+i \mid k)}$ is satisfied either. At time $k+2$, there are two cases which should be considered. Case 1: $\gamma(k+2 \mid k)=1, \gamma(k+1 \mid k)=1, \gamma(k \mid k)=1$; by applying (37), that is easy to be concluded $z(k+2 \mid k) \in \Theta_{\Delta(k+2 \mid k)}$. Case 2: $\gamma(k+2 \mid k)=1, \gamma(k+1 \mid k)=0, \gamma(k \mid k)=1$; assume $z(k+1 \mid$ $k) \in \Theta_{\Delta(k+1 \mid k)}:=\left\{z \in \Re^{n_{u}+n_{x}} \mid z^{T} \bar{U}_{0,1} z \leq 1\right\}$. By using the same method as above, it is obvious that LMIs (38) and (39) can guarantee the following inequalities, respectively,

$$
\begin{aligned}
& {\left[\begin{array}{cccc}
A_{i}+B_{i} \Lambda(k) K_{j} & 0 & -B_{i} & D_{i} \\
\Lambda(k) K_{j} & 0 & -1 & 0
\end{array}\right] \Xi_{0}^{1^{-1}}} \\
& \quad \times\left[\begin{array}{rrrr}
A_{i}+B_{i} \Lambda(k) K_{j} & 0 & -B_{i} & D_{i} \\
\Lambda(k) K_{j} & 0 & -1 & 0
\end{array}\right]^{T} \leq \bar{U}_{0,1}^{-1}, \\
& {\left[\begin{array}{cccc}
A_{i} & \theta B_{i} & 0 & D_{i} \\
0 & \theta & 0 & 0
\end{array}\right] \Xi_{0, c}^{-1}\left[\begin{array}{cccc}
A_{i} & \theta B_{i} & 0 & D_{i} \\
0 & \theta & 0 & 0
\end{array}\right]^{T} \leq \bar{U}_{1}^{-1},}
\end{aligned}
$$

which means $z(k+2 \mid k) \in \Theta_{\Delta(k+2 \mid k)}$. At time $k+i(i \geq 3)$, applying the similar approach, (37)-(40) guaranteed $z(k+i \mid$ $k) \in \Theta_{\Delta(k+i \mid k)}$.

Theorem 7. The hard constraints (5) can be guaranteed if the following LMIs exist:

$$
\bigwedge(p(k))\left[\begin{array}{ccc}
\bar{W} & * & * \\
\mho L_{i j}^{T} & \bar{M}_{1} & * \\
-1 & 0 & \bar{Q}
\end{array}\right] \geq 0
$$

where $\bar{W}=\operatorname{diag}\left\{\bar{u}_{1}^{2}, \bar{u}_{2}^{2}, \ldots, \bar{u}_{m}^{2}\right\}, \mho=\operatorname{diag}\left\{1+\delta_{1}, \ldots, 1+\delta_{m}\right\}$.

Proof. The hard constraints (5) can be written as $\mid u_{p}(k+i \mid$ $k) \mid \leq \bar{u}_{p}, i \geq 0$.Then, at time $k+1$, if data loss occurs, constraints (5) can be satisfied apparently. When the data is transmitted successfully at time $k+i$, by exploiting Theorem 5 , thus, we can obtain

$$
\begin{aligned}
u_{p}(k+i \mid k)^{2} & \left(\left(F_{j}^{T}\left(\Lambda(k+i) K_{j} x(k+i \mid k)-\phi(k)\right)\right)\right)^{2} \leq(1 \\
& +\omega(k+i \mid k))^{2} \| F_{j}^{T}\left[\Lambda(k+i) K_{j}-1\right] \\
& \times\left[\begin{array}{c}
x(k+i \mid k) \\
\phi(k)
\end{array}\right] \|^{2} \leq(1+\omega(k+i \mid k))^{2} \\
& \cdot\left[\begin{array}{cc}
F_{j}^{T}\left[\Lambda(k+i) K_{j}-1\right] \\
\quad
\end{array}\right]\left[\begin{array}{cc}
\bar{M}_{1}^{-1 / 2} & 0 \\
0 & \bar{Q}^{-1 / 2}
\end{array}\right]\left[\begin{array}{c}
x(k+i \mid k) \\
\phi(k)
\end{array}\right] \|^{2} \\
& \times\left[\begin{array}{cc}
\bar{M}_{1}^{1 / 2} & 0 \\
0 & \bar{Q}^{1 / 2}
\end{array}\right]\left[\begin{array}{cc}
\bar{M}_{1}^{1 / 2} & 0 \\
0 & \bar{Q}^{1 / 2}
\end{array}\right] \|^{2}
\end{aligned}
$$




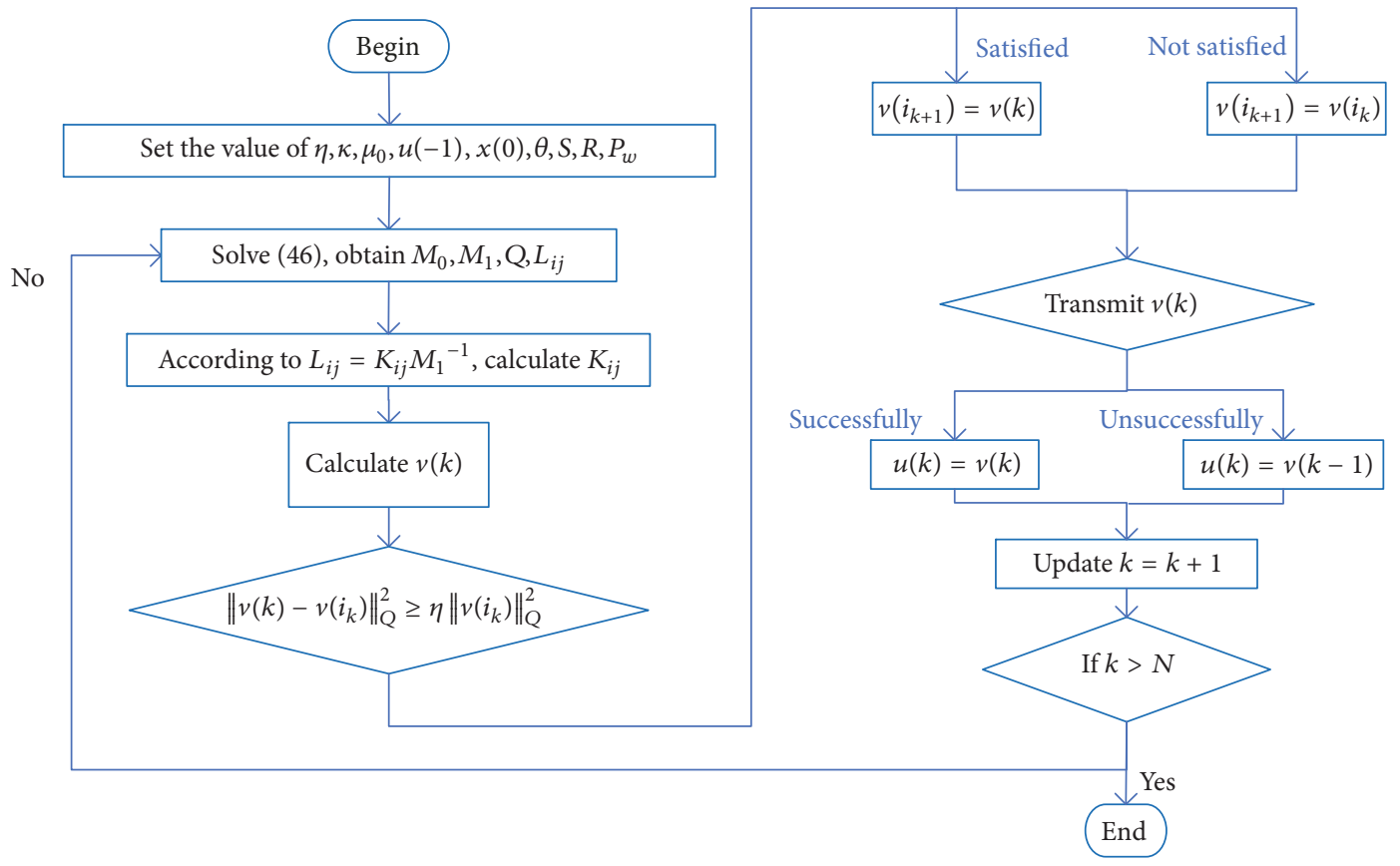

FIgURE 2: The completed procedure of Algorithm 1.

where $F_{j}$ is a j-column matrix that is selected from the mdimensional identity matrix. By using Schur complement, then (44) can be obtained.

Based on the above discussion, we have the following whole constrained predictive control optimization problem:

$$
\varepsilon, \overline{M_{0}}, \overline{N_{0}}, \overline{M_{1}}, \overline{N_{1}}, \overline{\mathrm{Q}}, \overline{E_{0}}, \overline{F_{0}}, \overline{E_{1}}, \overline{F_{1}}, L_{i j}, \bar{M}_{0, c}, \bar{N}_{0, c} \quad \mathcal{E}
$$

s.t.

$$
\text { (21), (23)-(26), (37), (38), (39), (40), and (44) }
$$

In summary, Algorithm 1 concludes the completed procedure of proposed method as Figure 2.

\subsection{Recursive Feasibility and Stochastic Stability of MPC}

Theorem 8. For augmented closed-loop system (17) controlled by state-feedback MPC, if the optimization problem (46) is feasible at time $k=k_{o}$, then there is a feasible solution for all future time. Moreover, the closed-loop system is stochastically stable by the feasible event-triggered predictive state-feedback control law in algorithm 1.

\section{Proof.}

Recursive Feasibility. According to the assumption, the augmented closed-loop system (17), since the state-feedback MPC optimization problem (46) is feasible at time $k_{o}$, we assume that the optimal solution is $\left\{\varepsilon\left(k_{o}\right)^{*}, L_{i j}\left(k_{o}\right)^{*}\right\}$. At time $k_{o}+1$, we can obtain a solution $\left\{\varepsilon\left(k_{o}\right)^{*}, L_{i j}\left(k_{o}\right)^{*}\right\}$; it is obvious that the new solution satisfies LMIs (23)-(26) and (37), (38), (39), (40), and (44) since the parameters are independent of the initial state $z(k)$. Hence, to prove recursive feasibility, we only need to prove the LMI (21) can be satisfied at time $k_{o}$. According to Theorem 5, we have $V\left(k_{o}+1 \mid k_{o}\right) \leq \varepsilon^{*}\left(k_{o}\right)$. Since the augmented state measured at $k_{o}+1$, it means $z\left(k_{o}+\right.$ $\left.1 \mid k_{o}+1\right)=z\left(k_{o}+1\right)=z\left(k_{o}+1 \mid k_{o}\right)$; it also needs to satisfy $V\left(k_{o}+1 \mid k_{o}+1\right)=V\left(k_{o}+1 \mid k_{o}\right) \leq \varepsilon^{*}\left(k_{o}\right)$. Then, at time $k_{o}+1$, the feasible solution is $\left\{\varepsilon\left(k_{0}\right)^{*}, L_{i j}\left(k_{0}\right)^{*}\right\}$. At time $k_{o}+2$, the data status have two conditions which should be considered. Case $1: 1 \longrightarrow 1$, that is, $\gamma\left(k_{o}\right)=1, \gamma\left(k_{0}+1\right)=$ 1. At time $k_{o}+1$, we assume that the optimal solution is $\left\{\varepsilon\left(k_{o}+1\right)^{*}, L_{i j}\left(k_{o}+1\right)^{*}\right\}$. At time $k_{o}+2$, by a similar analysis, we can obtain the feasible solution $\left\{\varepsilon\left(k_{o}+1\right)^{*}, L_{i j}\left(k_{o}+1\right)^{*}\right\}$. Case $2: 1 \longrightarrow 0$, that is, $\gamma\left(k_{o}\right)=1, \gamma\left(k_{0}+1\right)=0$. Since data are lost at time $k_{o}+1$, by solving optimization problem (46) and calculated by quantization and event-triggered scheme. We can get the control input at time $k_{o}+1$, but this data cannot be transmitted to the actuator successfully. Thus, the control input is dependent on the optimization problem at time $k_{o}$. According Theorem 5, we have $V\left(k_{o}+2 \mid k_{o}+1\right) \leq \varepsilon^{*}\left(k_{o}\right)$. For this data status, since the augment closed-loop system we can obtain $z\left(k_{o}+2 \mid k_{o}+2\right)=\left[\begin{array}{cccc}A_{i} & \theta B_{i} & B_{i} & D_{i} \\ 0 & \theta & 0 & 0\end{array}\right] z\left(k_{0}+1\right)=z\left(k_{o}+2 \mid\right.$ $\left.k_{o}\right)$; thus, it also can concluded that $V\left(z\left(k_{o}+2 \mid k_{o}+2\right)\right)=$ $V\left(k_{o}+2 \mid k_{o}\right) \leq \varepsilon^{*}\left(k_{o}\right)$. Furthermore, $\left\{\varepsilon\left(k_{o}+1\right)^{*}, L_{i j}\left(k_{o}+1\right)^{*}\right\}$ is a feasible solution at time $k_{o}+2$. At time $k_{o}+j(j \geq 3)$, by a similar analysis, the feasibility of optimization problem can be guaranteed.

Closed-Loop Stability. After above analysis, the recursive feasibility of (46) can be guaranteed; it means that the solution of (46) at time $k$ is also feasible at time $k+1$, which means $\varepsilon(k+1)=\varepsilon^{*}(k)$ is feasible for the optimization problem (46) at time $k+1$. Since $J(k)=\sum_{i=0}^{\infty} \mathbb{E}\|z(k+i \mid k)\|_{S}^{2}+\|u(k+i \mid k)\|_{R}^{2} \leq$ $\varepsilon(k)$, thus $\varepsilon^{*}(k)$ is decreasing and will converge to a little value with the increase of time $k$; it means $\{u\}$ will converge to a 

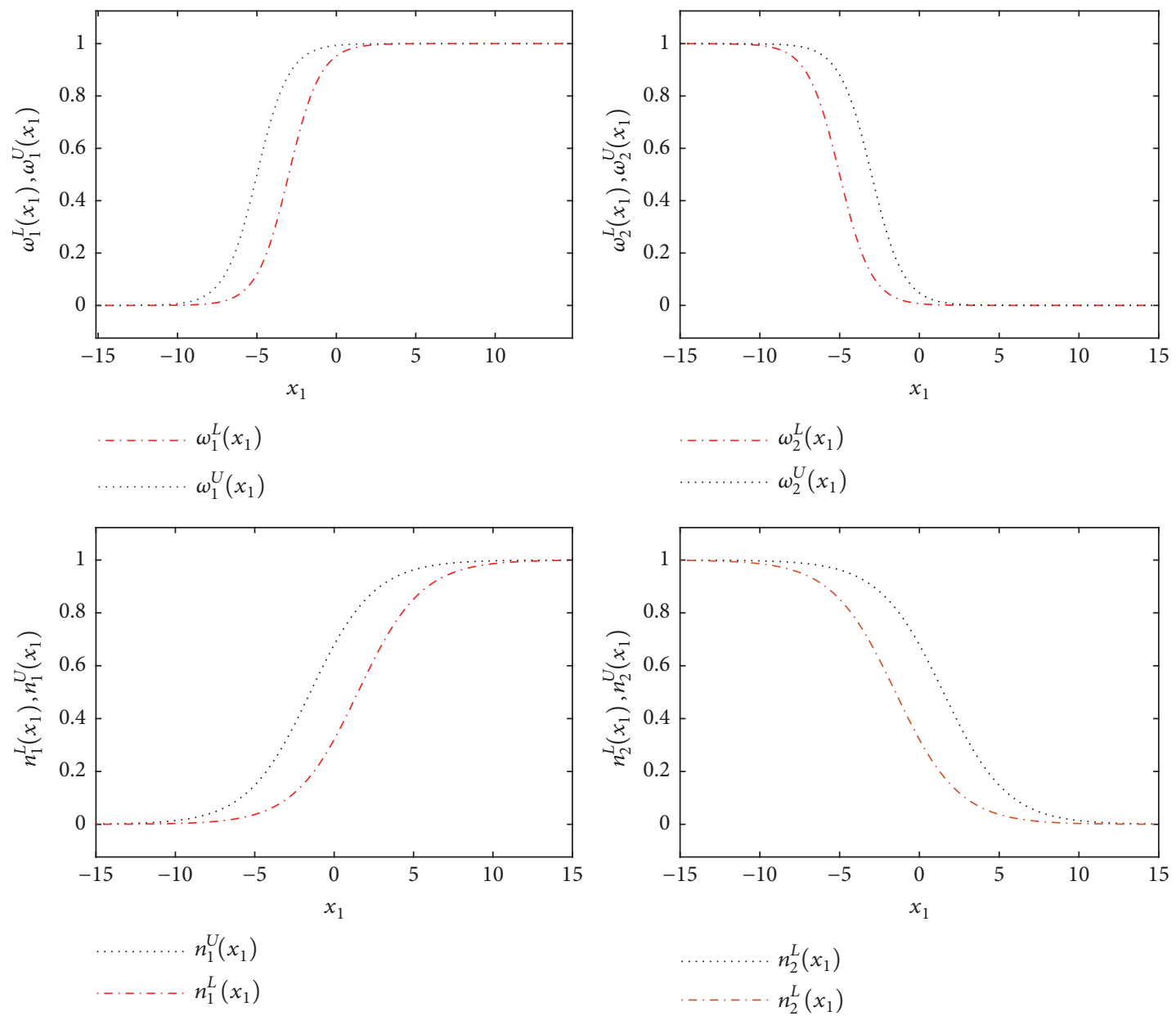

FiguRE 3: Membership functions.

neighborhood of $\{0\}$. Then the closed-loop stability can be proved.

\section{Simulations}

In this section, we proposed an example to demonstrate the merits of the presented method, and the figures in this simulation are reproduced from [37].

Consider that a 2-rule IT2 T-S fuzzy system and the matrices are given as follows:

$$
\begin{aligned}
& A_{1}=\left[\begin{array}{ccc}
1.05 & 0 & 0 \\
-0.05 & 1 & 0 \\
0.05 & -0.4 & 1
\end{array}\right], \\
& A_{2}=\left[\begin{array}{ccc}
1.05 & 0 & 0 \\
-0.05 & 1 & 0 \\
0.08 & -0.64 & 1
\end{array}\right], \\
& B_{1}=\left[\begin{array}{c}
-0.14 \\
0 \\
0
\end{array}\right],
\end{aligned}
$$

$$
\begin{aligned}
& B_{2}=\left[\begin{array}{c}
-0.14 \\
0 \\
0
\end{array}\right], \\
& D_{1}=\left[\begin{array}{c}
-0.01 \\
0 \\
0
\end{array}\right], \\
& D_{2}=\left[\begin{array}{c}
-0.01 \\
0 \\
0
\end{array}\right] .
\end{aligned}
$$

Define the membership functions for the IT2 T-S fuzzy system as follows:

$$
\begin{aligned}
& \omega_{1}^{L}\left(x_{1}\right)=1-\frac{1}{1+e^{x_{1}+4-1}} \\
& \omega_{1}^{U}\left(x_{1}\right)=1-\frac{1}{1+e^{x_{1}+4+1}} \\
& \omega_{2}^{L}\left(x_{1}\right)=1-\omega_{1}^{L}\left(x_{1}\right) \\
& \omega_{2}^{U}\left(x_{1}\right)=1-\omega_{1}^{U}\left(x_{1}\right)
\end{aligned}
$$



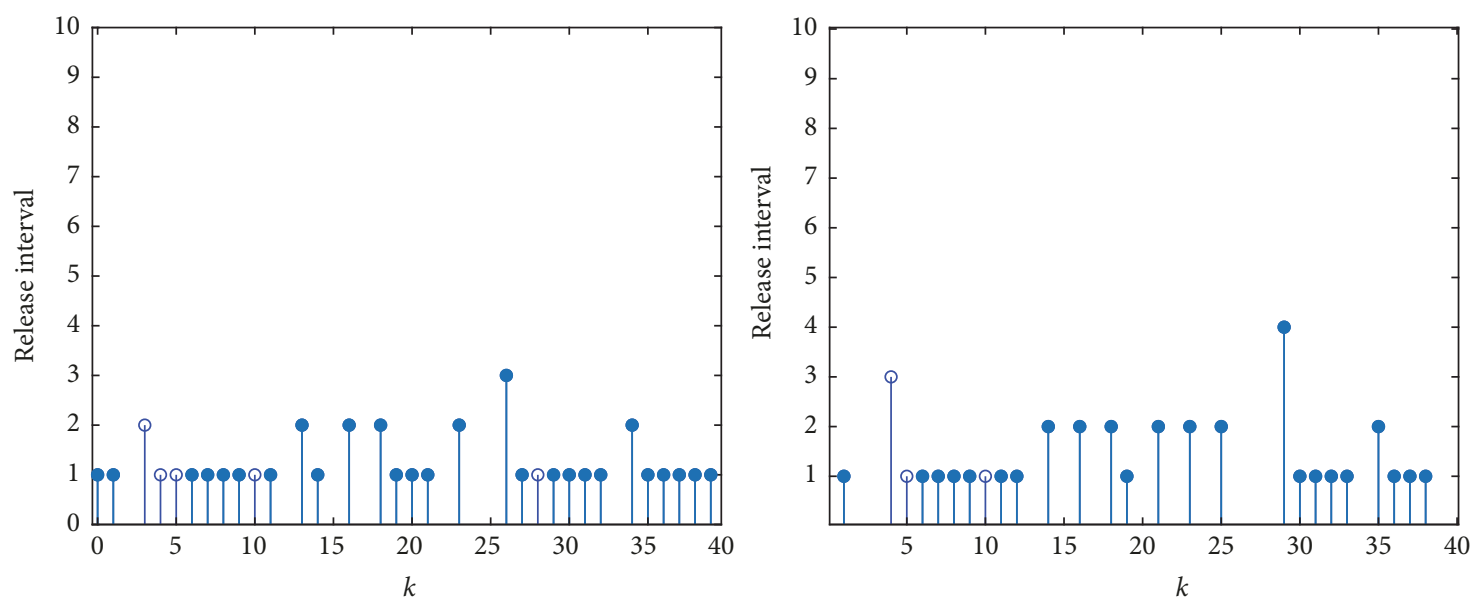

$\bigcirc \eta=0.015, \vartheta_{\max }=3$

$\bigcirc \eta=0.05, \vartheta_{\max }=3$
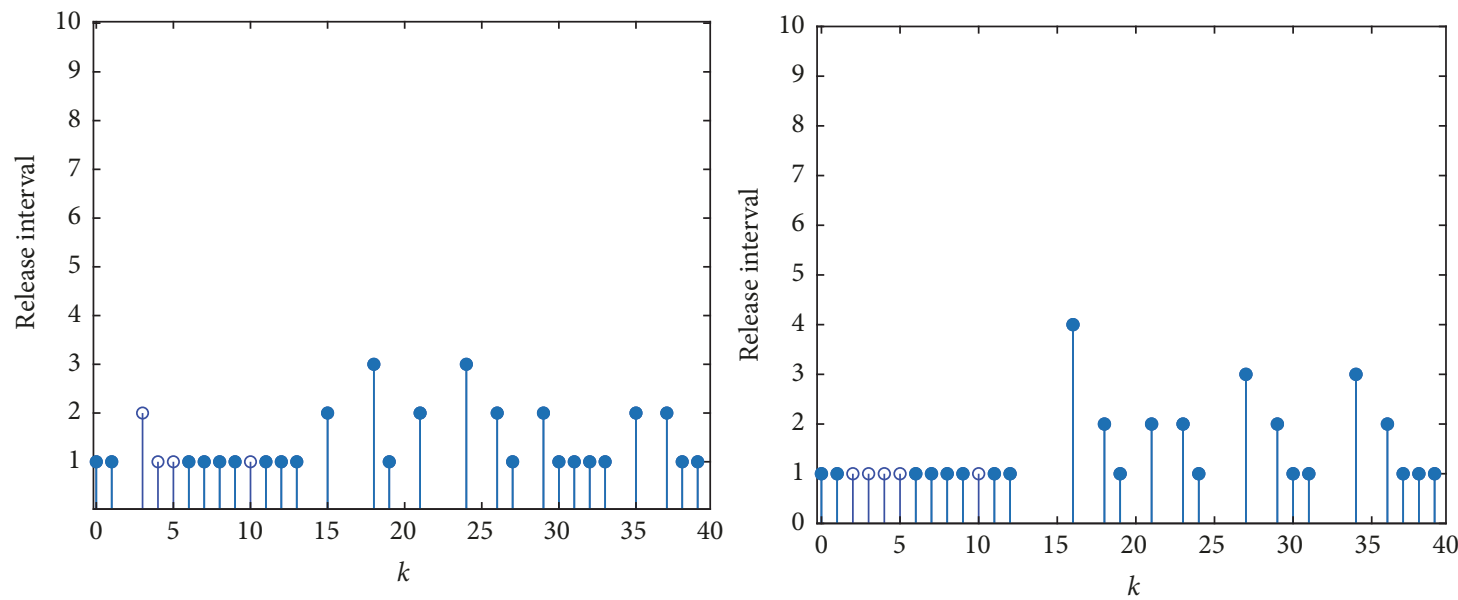

$\longrightarrow \eta=0.015, \vartheta_{\max }=5$

$\longrightarrow \eta=0.05, \vartheta_{\max }=5$

FIgURE 4: Release time and release interval with different the value of $\eta$ and $\vartheta_{\max }$.

$$
\begin{aligned}
& n_{1}^{L}\left(x_{1}\right)=1-\frac{1}{1+e^{-x_{1}-1.5}} \\
& n_{1}^{U}\left(x_{1}\right)=1-\frac{1}{1+e^{-x_{1}+1.5}} \\
& n_{2}^{L}\left(x_{1}\right)=1-\underline{n}_{1}^{L}\left(x_{1}\right) \\
& n_{2}^{U}\left(x_{1}\right)=1-\bar{n}_{1}^{U}\left(x_{1}\right)
\end{aligned}
$$

Define

$$
\begin{aligned}
& \bar{e}_{1}=\bar{h}_{1}=1-\sin ^{2}\left(x_{1}\right), \\
& \underline{e}_{1}=\underline{h}_{1}=\sin ^{2}\left(x_{1}\right), \\
& \bar{e}_{2}=\bar{h}_{1}=1-\cos ^{2}\left(x_{1}\right), \\
& \underline{e}_{2}=\underline{h}_{2}=\cos ^{2}\left(x_{1}\right) .
\end{aligned}
$$

Assume $x_{1}(k) \in[-15,15]$; it is related to the membership functions; Figure 3 shows the lower and upper membership

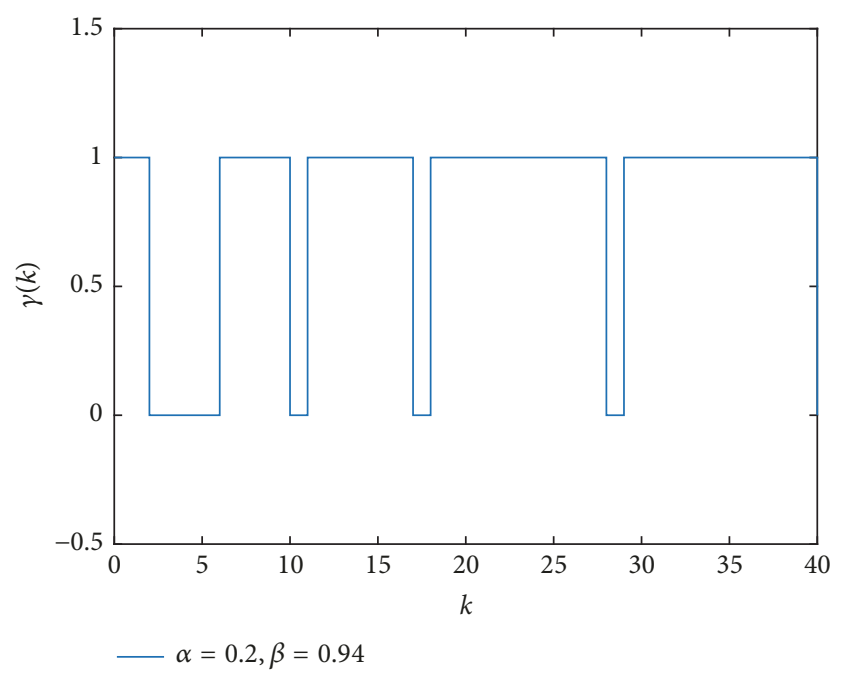

FIGURE 5: The data status.

functions. Assume the logarithmic quantizer parameters as $\mu_{0}=5, \rho=0.9608$. Figure 5 represents the Markov chain 

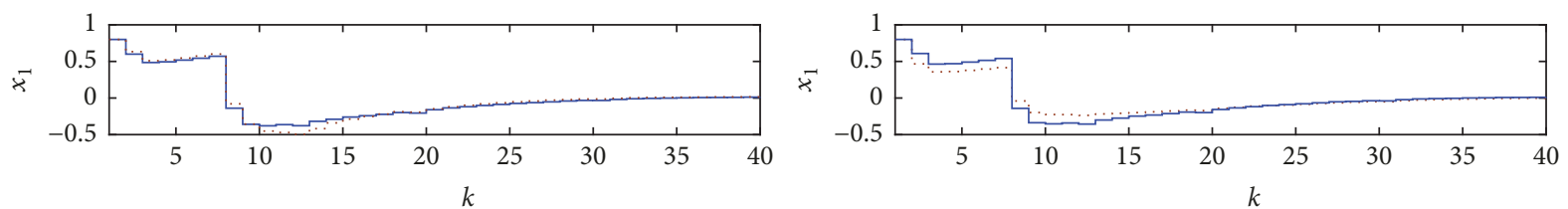

$$
\begin{aligned}
\eta & =0.015, \vartheta_{\max }=5 \\
\cdots . \eta & =0.015,9_{\max }=3
\end{aligned}
$$

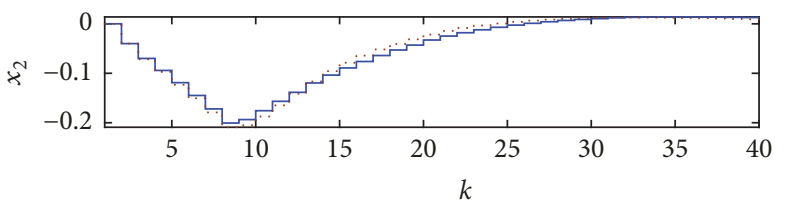

$$
\begin{aligned}
\cdots \cdots \eta & =0.05, \vartheta_{\max }=5 \\
\smile \eta & =0.025, \vartheta_{\max }=5
\end{aligned}
$$

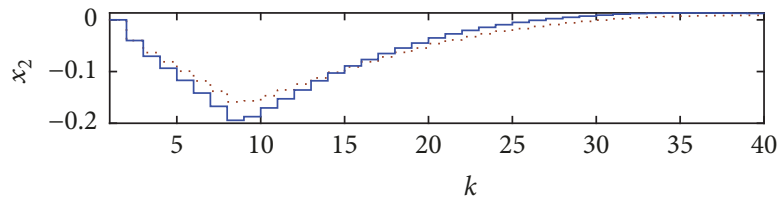

$$
\begin{aligned}
\eta & =0.015, \vartheta_{\text {max }}=5 \\
\cdots \cdots \eta & =0.015, \vartheta_{\text {max }}=3
\end{aligned}
$$

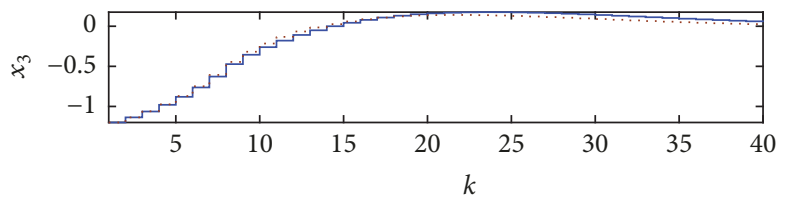

$$
\begin{aligned}
\cdots \cdots \eta & =0.05, \vartheta_{\max }=5 \\
-\eta & =0.025, \vartheta_{\max }=5
\end{aligned}
$$

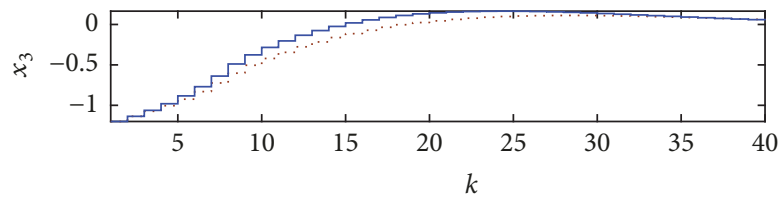

$$
\begin{aligned}
\eta & =0.015, \vartheta_{\max }=5 \\
\eta & =0.015, \vartheta_{\max }=3
\end{aligned}
$$

$$
\begin{aligned}
\cdots \cdots & =0.05, \vartheta_{\text {max }}=5 \\
-\eta & =0.025, \vartheta_{\text {max }}=5
\end{aligned}
$$

FIGURE 6: State responses.
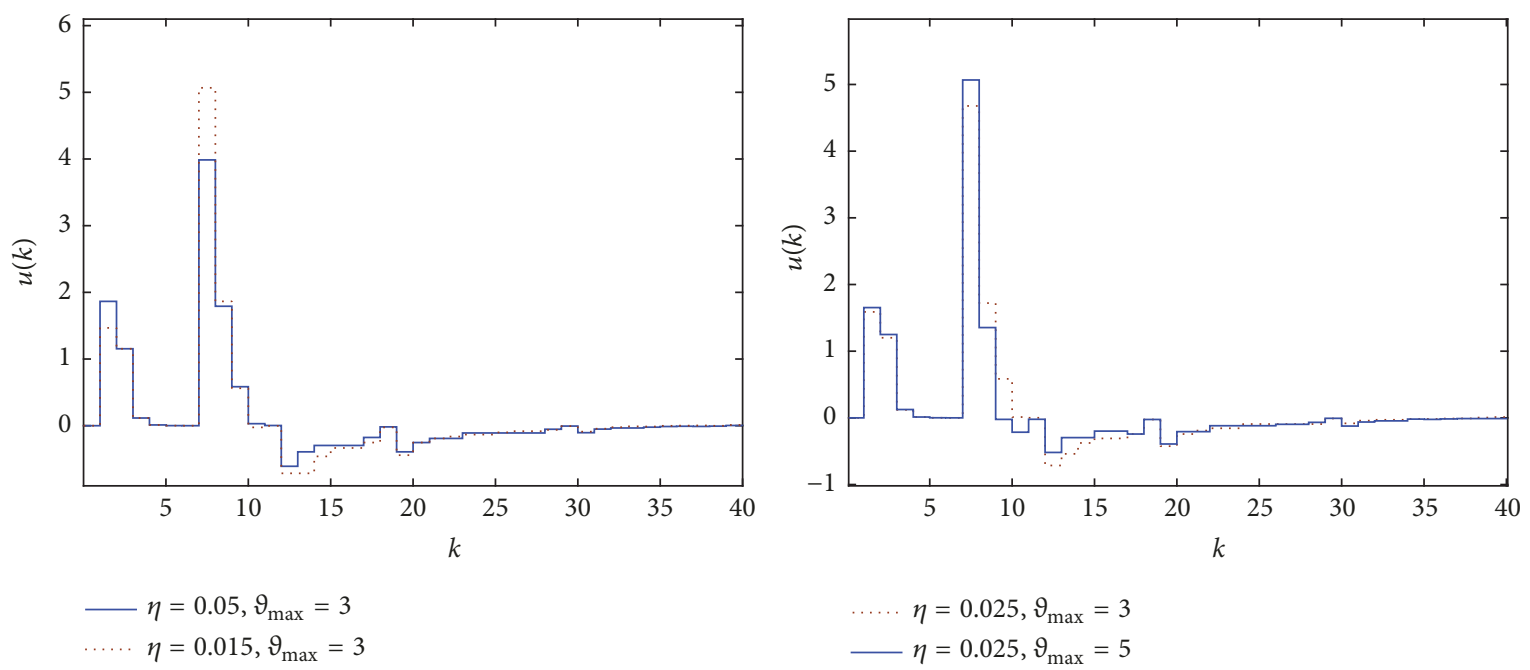

FIGURE 7: Control inputs.

data status and with the transition probability matrix $\Omega=$ $\left[\begin{array}{cc}0.06 & 0.94 \\ 0.2 & 0.8\end{array}\right], \alpha=0.2, \beta=0.94$. Assume that the upper of control input is $\bar{u}=10$ and the bounded disturbance $w(k) \in$ $[-0.10,1]$ is randomly generated. Define the weighting matrices of performance objective $\mathrm{S}=I_{3}, R=0.1, P_{w}=$ $100, \kappa=0.01$. Set the initial condition $x_{0}=\left[\begin{array}{lll}0.8 & 0 & -1.2\end{array}\right]^{T}$ and initial event-triggered control input as $u(-1)=0$, and the forgetting factor is $\theta=0.1$.

From Remark 1, we consider the two cases of $\eta$ with same $\vartheta_{\max }$ to prove that the value of $\eta$ would affect the eventtriggered frequency. By applying the proposed algorithm, we can obtain the following results. Figure 4 shows the event-triggered release time and release interval with the different value of $\eta$; from the picture we can see that the bigger $\eta$ is, the smaller the event-triggered times are. Moreover, not all triggered data will be transmitted into the actuator due to the unreliable communication network. In Figure 4, the filled circles represent that the event-triggered data released into the network successfully. The control inputs received by the actuator are shown in Figure 7; it is shown that the control input will converge to a neighborhood of $u=0$ under the event-triggered scheme regardless of the influences of quantization error and data loss; furthermore, the input constraint can be satisfied either. The state responses of states are depicted in Figure 6; this figure shows the closed-loop system is stable by exploiting the event-triggered state-feedback 

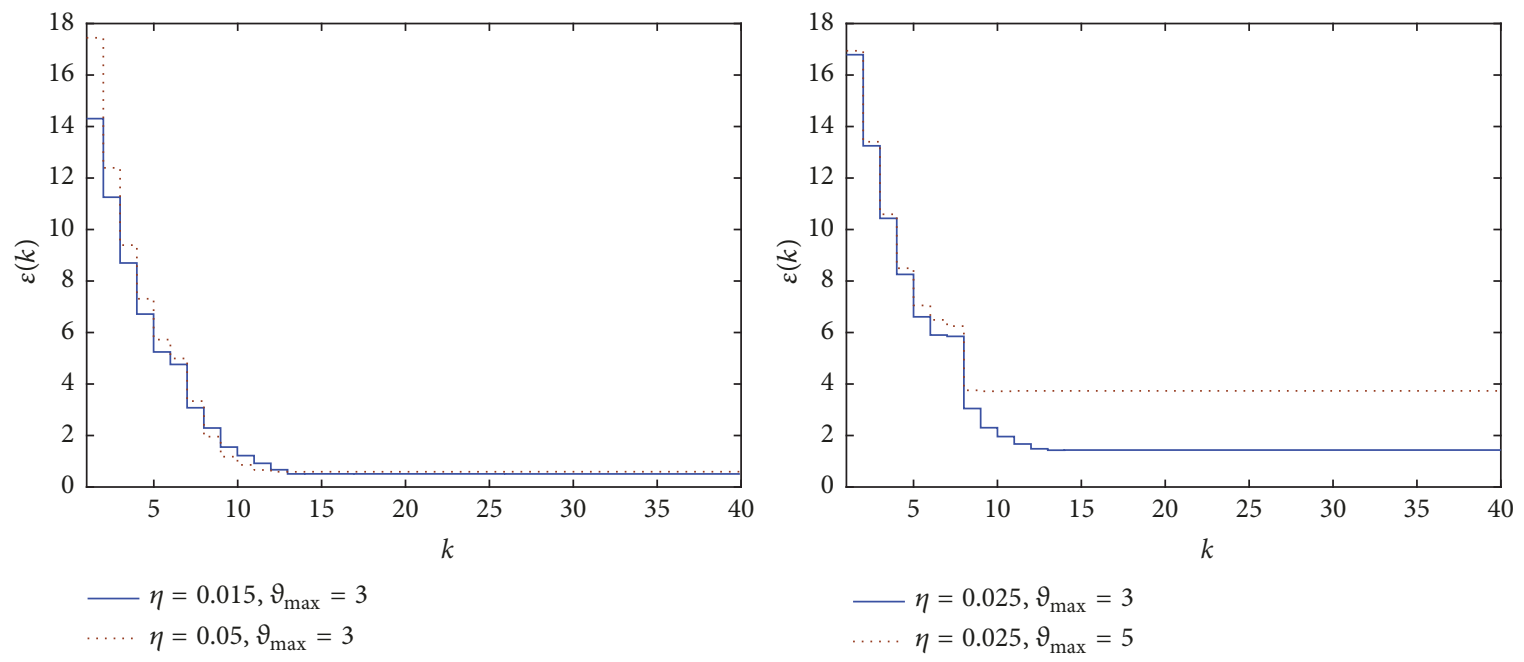

FIGURE 8: Evolutions of $\varepsilon(k)$.

MPC method. The upper $\varepsilon(k)$ of control performance is shown in Figure 8.

\section{Conclusion}

This paper provides the event-triggered constrained predictive control for IT2 T-S fuzzy system with data loss and bounded disturbance. A novel event-triggered scheme is applied to decide whether the controller output should be released into the unreliable communication network or not. The phenomenon of data loss occurs between the transition from the controller to the actuator is described as Markovian data loss model. In order to design the statefeedback controller, we proposed an online optimization problem which minimizes the upper bound of quadratic objective function in an infinite time horizon subjected to input constraint which can be solved by LMI technique at each time $k$. The effectiveness of this approach is proved by the simulation example. In the future, an improvement of this approach would consider the problem of time delays problem in communication networks or using multistep algorithm to achieve better control performance.

\section{Data Availability}

The data used to support the findings of this study are available from the corresponding author upon request.

\section{Conflicts of Interest}

The authors declare that they have no conflicts of interest.

\section{Acknowledgments}

This work is supported by the Research Project of Chongqing Science and Technology Commission (cstc2018jcyjAX0691), the Scientific and Technological Research Program of Chongqing Municipal Education
Commission (KJQN201800645), and the Scientific and Technological Research Program of Chongqing Municipal Education Commission (KJQN201803110).

\section{References}

[1] Y. Niu and D. W. Ho, "Design of sliding mode control subject to packet losses," IEEE Transactions on Automatic Control, vol. 55, no. 11, pp. 2623-2628, 2010.

[2] P. Lin and W. Ren, "Constrained consensus in unbalanced networks with communication delays," IEEE Transactions on Automatic Control, vol. 59, no. 3, pp. 775-781, 2014.

[3] D. Q. Truong and K. K. Ahn, "Robust Variable Sampling Period Control for Networked Control Systems," IEEE Transactions on Industrial Electronics, vol. 62, no. 9, pp. 5630-5643, 2015.

[4] L. A. Montestruque and P. J. Antsaklis, "On the model-based control of networked systems," Automatica, vol. 39, no. 10, pp. 1837-1843, 2003.

[5] P. Lin, C. Lin, C. Hsu, and T. Lee, “Type-2 fuzzy controller design using a sliding-mode approach for application to DC-DC converters," IEE Proceedings - Electric Power Applications, vol. 152, no. 6, pp. 1482-1488, 2005.

[6] H. A. Hagras, "A hierarchical type-2 fuzzy logic control architecture for autonomous mobile robots," IEEE Transactions on Fuzzy Systems, vol. 12, no. 4, pp. 524-539, 2004.

[7] P. Shi, H. Wang, and C.-C. Lim, "Network-based eventtriggered control for singular systems with quantizations," IEEE Transactions on Industrial Electronics, vol. 63, no. 2, pp. 12301238, 2016.

[8] P. Seiler and R. Sengupta, "An Ho approach to networked control," Institute of Electrical and Electronics Engineers Transactions on Automatic Control, vol. 50, no. 3, pp. 356-364, 2005.

[9] C.-H. Fang, Y.-S. Liu, S.-W. Kau, L. Hong, and C.-H. Lee, "A new LMI-based approach to relaxed quadratic stabilization of T-S fuzzy control systems," IEEE Transactions on Fuzzy Systems, vol. 14, no. 3, pp. 386-397, 2006.

[10] Y. Zhao, H. Gao, J. Lam, and B. Du, "Stability and stabilization of delayed T-S fuzzy systems: a delay partitioning approach," IEEE Transactions on Fuzzy Systems, vol. 17, no. 4, pp. 750-762, 2009. 
[11] A. S. S. Abadi, M. H. Mehrizi, and P. A. Hosseinabadi, "Fuzzy adaptive terminal sliding mode control of SIMO nonlinear systems with T-S fuzzy model," in Proceedings of the 6th Iranian Joint Congress on Fuzzy and Intelligent Systems, CFIS 2018, pp. 185-189, Kerman, Iran, March 2018.

[12] M. A. Sancheza, O. Castillo, and J. R. Castroa, "Information granule formation via the concept of uncertainty-based information with Interval Type-2 Fuzzy Sets representation and Takagi-Sugeno-Kang consequents optimized with Cuckoo search," Applied Soft Computing, vol. 27, pp. 602-609, 2015.

[13] E. Ontiveros-Robles, P. Melin, and O. Castillo, "Comparative analysis of noise robustness of type 2 fuzzy logic controllers," Kybernetika, vol. 54, no. 1, pp. 175-201, 2018.

[14] O. Castillo, L. Amador-Angulo, J. R. Castro, and M. GarciaValdez, "A comparative study of type-1 fuzzy logic systems, interval type-2 fuzzy logic systems and generalized type-2 fuzzy logic systems in control problems," Information Sciences, vol. 354, pp. 257-274, 2016.

[15] T. Zhao and S. Dian, "State feedback control for interval type-2 fuzzy systems with time-varying delay and unreliable communication links," IEEE Transactions on Fuzzy Systems, vol. 26, no. 2, pp. 951-966, 2018.

[16] L. Cervantes and O. Castillo, "Type-2 fuzzy logic aggregation of multiple fuzzy controllers for airplane flight control," Information Sciences, vol. 324, pp. 247-256, 2015.

[17] O. Castillo, L. Cervantes, J. Soria, M. Sanchez, and J. R. Castro, "A generalized type-2 fuzzy granular approach with applications to aerospace," Information Sciences, vol. 354, pp. 165-177, 2016.

[18] H. Y. Li, C. W. Wu, P. Shi, and Y. B. Gao, "Control of nonlinear networked systems with packet dropouts: interval type-2 fuzzy model-based approach," IEEE Transactions on Cybernetics, vol. 45, no. 11, pp. 2378-2389, 2015.

[19] H. Y. Li, C. W. Wu, L. G. Wu, H. K. Lam, and Y. B. Gao, "Filtering of interval type-2 fuzzy systems with intermittent measurements," IEEE Transactions on Cybernetics, vol. 46, no. 3, pp. 668-678, 2016.

[20] L. Ding, Q. Han, X. Ge, and X. Zhang, "An overview of recent advances in event-triggered consensus of multiagent systems," IEEE Transactions on Cybernetics, vol. 48, no. 4, pp. 1110-1123, 2018.

[21] C. Peng and T. C. Yang, "Event-triggered communication and Ho control co-design for networked control systems," Automatica, vol. 49, no. 5, pp. 1326-1332, 2013.

[22] C. Peng, Q.-L. Han, and D. Yue, "To transmit or not to transmit: a discrete event-triggered communication scheme for networked takagi-sugeno fuzzy systems," IEEE Transactions on Fuzzy Systems, vol. 21, no. 1, pp. 164-170, 2013.

[23] C. Peng, M. Wu, X. Xie, and Y. Wang, "Event-triggered predictive control for networked nonlinear systems with imperfect premise matching," IEEE Transactions on Fuzzy Systems, vol. 26, no. 5, pp. 2797-2806, 2018.

[24] C. Peng, S. Ma, and X. Xie, “Observer-based non-PDC control for networked T-S fuzzy systems with an event-triggered communication," IEEE Transactions on Cybernetics, vol. 47, no. 8, pp. 2279-2287, 2017.

[25] L. Zhang, J. Wang, Y. Ge, and B. Wang, "Robust distributed model predictive control for uncertain networked control systems," IET Control Theory \& Applications, vol. 8, no. 17, pp. 1843-1851, 2014.

[26] H. Han, X. Zhang, and W. Zhang, "Robust distributed model predictive control under actuator saturations and packet dropouts with time-varying probabilities," IET Control Theory \& Applications, vol. 10, no. 5, pp. 534-544, 2016.

[27] Z. Pang, G. Liu, and D. Zhou, "Design and performance analysis of incremental networked predictive control systems," IEEE Transactions on Cybernetics, vol. 46, no. 6, pp. 1400-1410, 2016.

[28] J. Zhang, Y. Xia, and P. Shi, "Design and stability analysis of networked predictive control systems," IEEE Transactions on Control Systems Technology, vol. 21, no. 4, pp. 1495-1501, 2013.

[29] J. Li, D. Li, and Y. Xi, "Multi-step probabilistic sets in model predictive control for stochastic systems with multiplicative uncertainty," IET Control Theory \& Applications, vol. 8, no. 16, pp. 1698-1706, 2014.

[30] D. Li, Y. Xi, and F. Gao, "Synthesis of dynamic output feedback robust model predictive control with saturated inputs," Automatica, vol. 49, no. 4, pp. 949-954, 2013.

[31] M. Lješnjanin, D. E. Quevedo, and D. Nešić, "Packetized MPC with dynamic scheduling constraints and bounded packet dropouts," Automatica, vol. 50, no. 9, pp. 784-797, 2014.

[32] J. Lu, D. Li, and Y. Xi, "Constrained model predictive control synthesis for uncertain discrete-time Markovian jump linear systems," IET Control Theory \& Applications, vol. 7, no. 5, pp. 707-719, 2013.

[33] X. M. Tang and B. C. Ding, "Model predictive control of linear systems over networks with data quantizations and packet losses," Automatica, vol. 49, no. 5, pp. 1333-1339, 2013.

[34] Y. Zou, J. Lam, Y. Niu, and D. Li, "Constrained predictive control synthesis for quantized systems with Markovian data loss," Automatica, vol. 55, pp. 217-225, 2015.

[35] M. Fu and L. Xie, "The sector bound approach to quantized feedback control," IEEE Transactions on Automatic Control, vol. 50, no. 11, pp. 1698-1711, 2005.

[36] A. Alessandri, M. Baglietto, and G. Battistelli, "On estimation error bounds for receding-horizon filters using quadratic boundedness," IEEE Transactions on Automatic Control, vol. 49, no. 8, pp. 1350-1355, 2004.

[37] X. Tang and S. Wang, "Event-triggered constrained predictive synthesis for interval type-2 fuzzy system with markovian data loss," in Proceeding of the 2018 Chinese Automation Congress (CAC), pp. 1536-1541, Xi'an, China, 2018. 


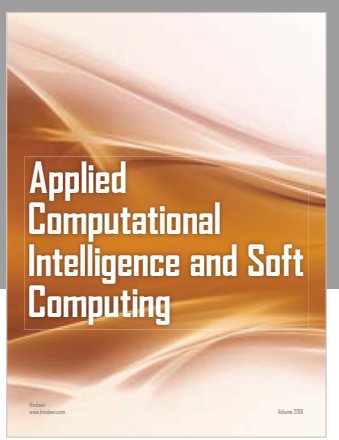

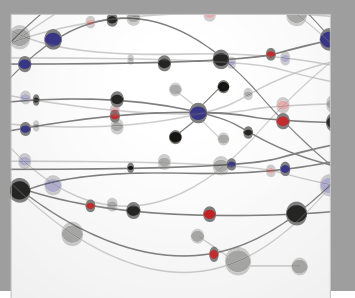

The Scientific World Journal
Submit your manuscripts at

Computing
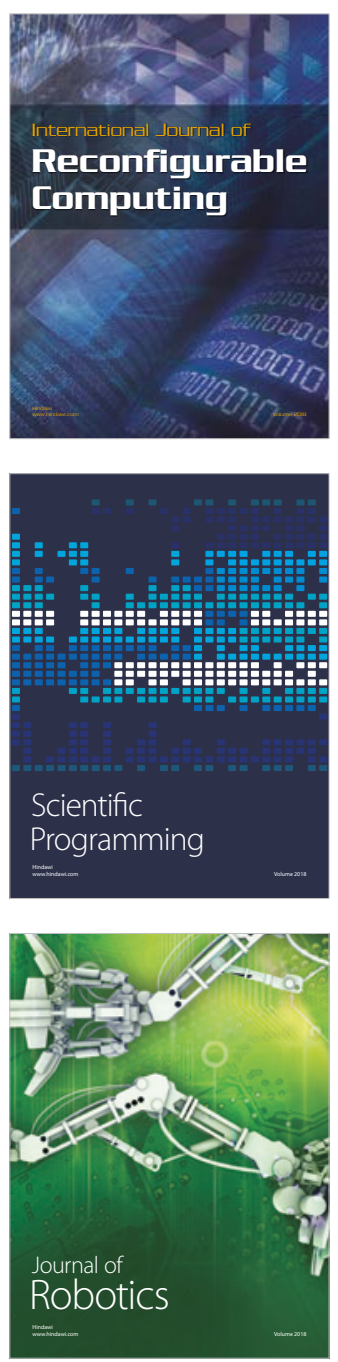

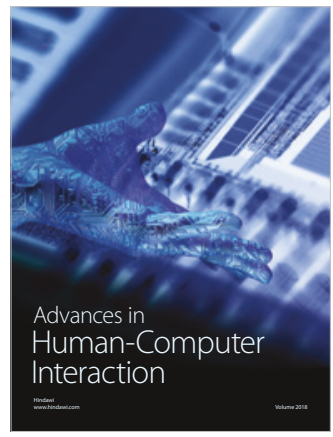

Human-Compute

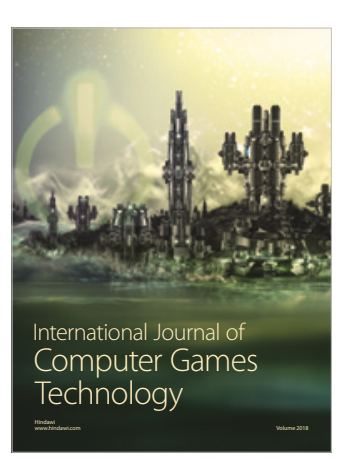

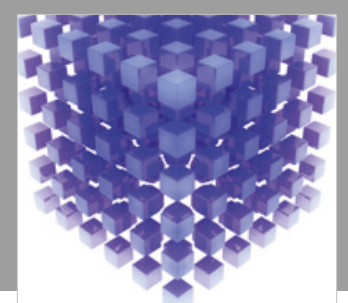

Mathematical Problems in Engineering

\section{Engincering}
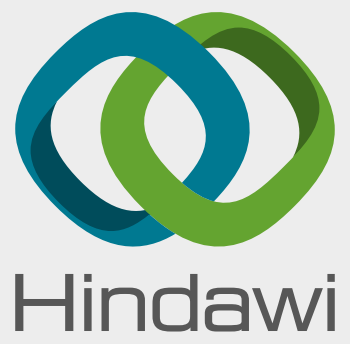

www.hindawi.com
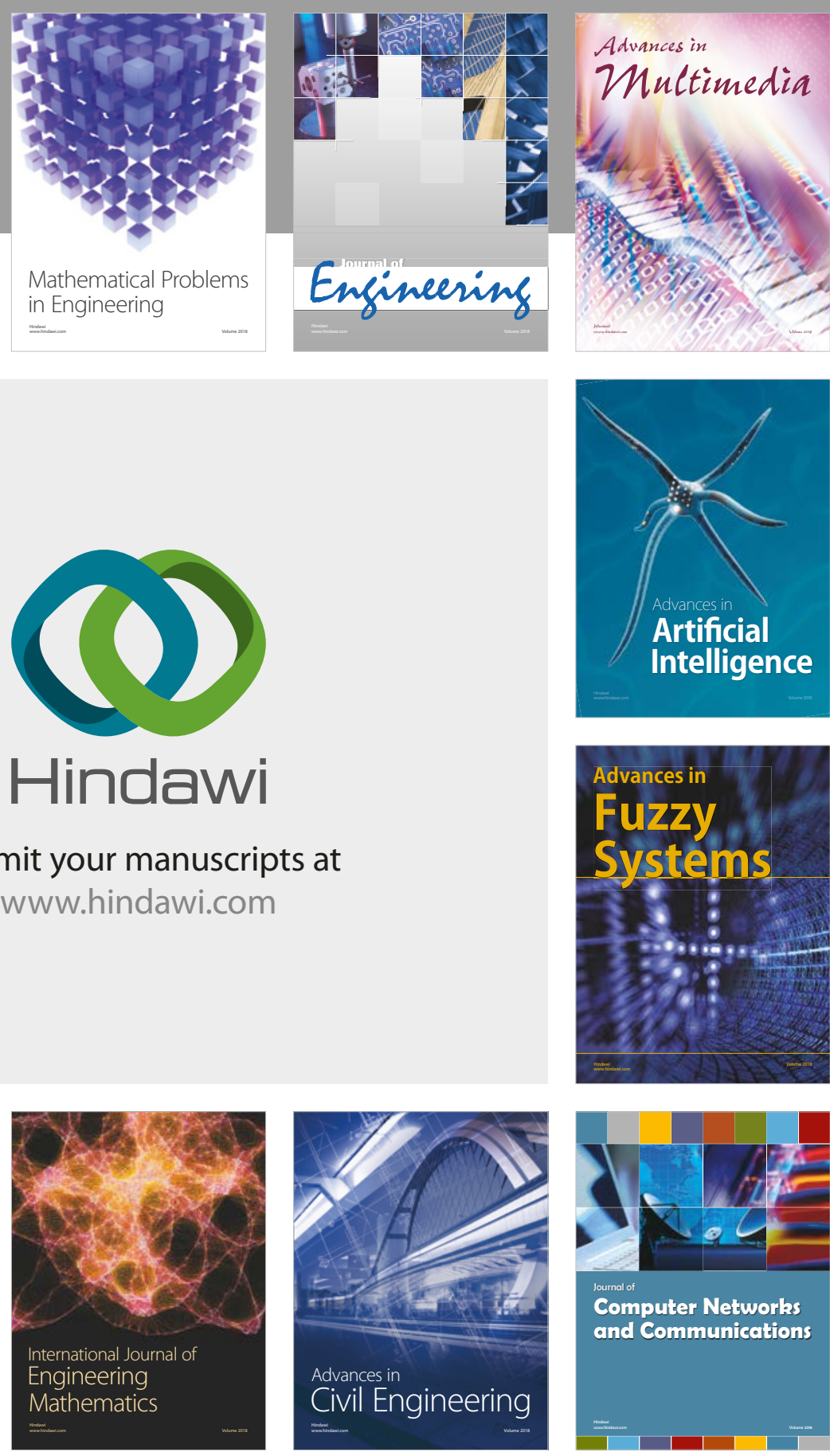

Computer Networks and Communications

Multimedia
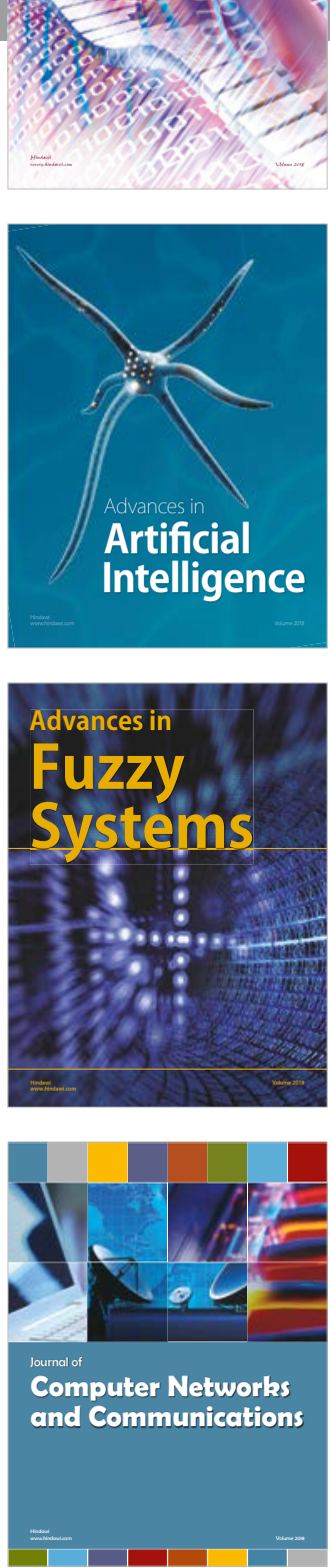

Advances in

Modelling \&

Simulation

in Engineering

interaction

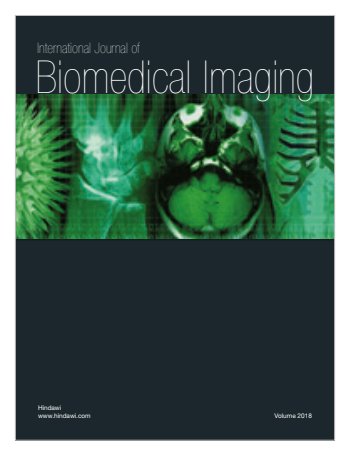

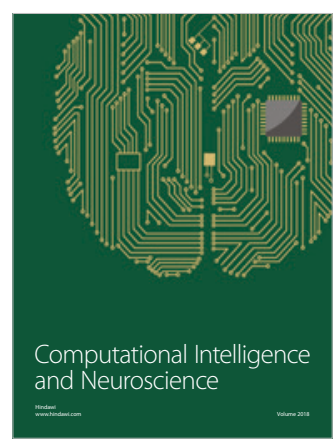

